\title{
Angstgefühle und deren mögliche Auswirkungen auf das Lernen von Fremdsprachen. Fokus: Studienanfänger/innen in der Germanistik - eine Fallstudie
}

W badaniach glottodydaktycznych nad akwizycją języka obcego ważną rolę odgrywają cechy indywidualne uczących się, które niejednokrotnie determinują procesy uczeniowe. W tym kontekście badaczy interesuje zjawisko lęku językowego, źródeł jego powstawania i wpływu na komunikację obcojęzyczną. Niniejszy artykuł ma charakter analizy empirycznej i odnosi się do badań ankietowych przeprowadzonych wśród studentów I roku kierunków magisterskich i zawodowych na filologii germańskiej. Respondenci odnosili się do swoich wcześniejszych doświadczeń szkolnych. Za narzędzie badawcze posłużyła standardowa ankieta opracowana przez HoRwITz / HoRwITz / COPE (1986). Wyniki badań nie wykazały, iż obie grupy zasadniczo róźnią się pomiędzy sobą poziomem lęku. Róźnice można zaobserwować jedynie w pojedynczych aspektach. Wyciagnięte wnioski mogą posłużyć jako pomoc przy organizowaniu pracy dydaktycznej na zajęciach praktycznej nauki języka w szkole wyższej.

Angst ist unzweifelhaft einer der individuellen Faktoren, die das Lernen einer Fremdsprache überwiegend beeinträchtigen. In diesem Beitrag wird eine empirische Analyse vorgestellt, in der die Studienanfänger/innen in Germanistik nach ihren Schulerfahrungen beim Deutschlernen gefragt wurden. Die Daten wurden mit einem standardisierten Fragebogen von HoRwITZ / HORwITZ / COPE (1986) erhoben. Untersucht wurden zwei Gruppen - Magisterstudierende und Berufsstudierende. Die Ergebnisse haben keine signifikanten Unterschiede zwischen den Gruppen ergeben, wobei sich in einigen Aspekten doch Differenzen beobachten lassen. Die Diskussion bezieht sich auf die möglichen Ursachen der Angstentstehung und didaktische Möglichkeiten, wie man den hinderlichen Angstgefühlen entgegenwirken kann. 
Krzysztof Nerlicki

In glottodidactic research into second language acquisition, an important role is played by the learner's individual features as they usually determine the learning process. In this context, researchers are particulary interested in the anxiety of speaking experienced by many learners, its origins and influence on the process of communication in a foreign language. This article is an empirical study that presents results of a questionnaire completed by the first-year German philology students of BA and MA studies. While answering the questions, the respondents referred to their earlier school experiences. A standard questionnaire described in HORWITZ / HORWITZ / COPE (1986) was used in this particular research. The results did not show significant differences between the level of anxiety experienced by both groups. There are, however, differences in some aspects. The obtained results, and the conclusions that can be drawn from them, can be used in preparing practical language classes for college students.

\section{Vorbemerkungen}

Die moderne Fremdsprachenlehr- und -lernforschung widmet sich zunehmend den individuellen Faktoren, die die Lern- und Kommunikationsprozesse, insbesondere im institutionellen Kontext, beeinflussen (können). Dies resultiert aus dem Perspektivenwechsel, den diese Fachdisziplin in den letzten Jahrzehnten durchlaufen hat und gemäß dem die Lernenden als Individuen betrachtet werden, was auch im Großen und Ganzen individuelle Lernwege impliziert. Das Forschungsinteresse wird u. a. auf kognitive und emotionale individuelle Unterschiede gerichtet, wie Geschlecht, Intelligenz, Sprachlerneignung, Lernstile und -strategien, Motivation, Einstellungen, Persönlichkeitsmerkmale und Alter (vgl. zur Übersicht EDMONDSON / HOUSE 1993, DÖRNYEI / SKEHAN 2003). In diesem Sinne wird auch der Angst im Kontext fremdsprachlicher Lehr- und Lernprozesse immer mehr Aufmerksamkeit geschenkt. Es ist nicht zu leugnen, dass dieser Faktor im Alltagsleben negative Assoziationen weckt. Aus fremdsprachendidaktischer Sicht erscheint die Frage legitim, was den Angstgefühlen zugrunde liegt und wie sich dies auf das Lernen und Kommunizieren auswirken kann. Die folgende Studie hat empirischen, fallorientierten Charakter. Untersucht wird, ob die polnischen Studienanfänger/innen in der Germanistik mit ihrem bisherigen schulischen Deutschlernen Angstgefühle verbinden und welche Angstgefühle das sind. Dazu wurden zwei Gruppen ausgewählt: Studierende im fünfjährigen Magisterstudium und Studierende im dreijährigen Berufsstudium. Im ersten Kapitel wird über den bisherigen Forschungsstand berichtet. Dann folgt die Beschreibung der empirischen Untersuchung und ihrer Ergebnisse. Im dritten Abschnitt schließt sich eine Diskussion aus erwerbsspezifischer und didaktischer Perspektive an. Zuletzt wird ausblickartig auf einige bleibende Probleme hingewiesen. 


\section{Angst und ihre Erscheinungsformen beim Fremdsprachenlernen und -gebrauch}

Das Wort Angst leitet sich vom lateinischen , angustia ' ab und bedeutet so viel wie Enge. Angst als affektiver Faktor kann als generelles Persönlichkeitsmerkmal (,trait anxiety', auch Ängstlichkeit genannt) oder aber als situationsspezifische Erscheinung im menschlichen Verhalten (,state anxiety') auftreten. HACKFORT / SCHWENKMEZGER (1985:19) positionieren die Angst im Bereich kognitiver, emotionaler und körperlicher Reaktionen auf eine Gefahrensituation bzw. deren Erwartung. Sie lässt sich also an vegetativ-somatischen Symptomen (z.B. Herzklopfen, Bauchschmerzen, Schwitzen, Muskelverspannung), psychischen Zuständen (z.B. Unsicherheit, Schüchternheit, Überempfindlichkeit, Panik) sowie an Verhaltensformen erkennen (z. B. Sprechstörungen, Stottern, Scherzen, nervöses Lachen, Grimassen, kein Blickkontakt; BEUSHAUSEN 1996). Aus kognitiver Sicht beeinträchtigen die Angstgefühle unsere emotionalen Ressourcen, so dass sie nicht mehr für die Lernprozesse zur Verfügung stehen (LEAVER / EHRMANN / SHEKTMAN 2005:93). HORWITZ / HORWITZ / COPE (1986:128) verstehen fremdsprachliche Angst als ,,[...] a distinct complex of self-perceptions, beliefs, feelings, and behaviors related to classroom language learning arising from the uniqueness of the learning language process". Aus der Definition wird ersichtlich, dass fremdsprachliche Angst als Konstrukt von mehreren Faktoren verstanden wird. Sie reichen von objektiven/subjektiven Selbstwahrnehmungen und Selbstkonzepten über erfahrungsbasierte Überzeugungen und Gefühle bis hin zu beobachtbaren Verhaltensweisen. Zu klären ist, in welchem Grade die Angst, die sich im Fremdsprachenunterricht oder in fremdsprachlicher Kommunikation beobachten lässt, ihre Grundlage in der Spezifik des Faches und/oder in anderen Größen (allgemeine Schulangst, Angst als Persönlichkeitsmerkmal) haben kann. Darüber hinaus ist die Frage berechtigt, welche Auswirkungen Angstgefühle auf das Lernen einer Fremdsprache haben können und ob (wenn vorhanden) sie sich im Unterricht vermindern lassen. HORWITZ / HORWITZ / COPE (1986) haben einen der bekanntesten Fragebögen zur Messung von Angstfaktoren im Fremdsprachenunterricht - „Foreign Language Classroom Anxiety Scale (FLCAS)“ - entwickelt. ${ }^{1}$ Der Fragebogen enthält 33 Items. Die

Die entwickelten Instrumente zur Messung der Angst berücksichtigen sehr oft die lokale Spezifik des Fremdsprachenlernens. So haben wir z. B. die „French Class Anxiety Scale“ und die „English Use Anxiety Scale“ (nach GARDNER / TrEmBlay / MASGORET 1997). „FLCAS“ ist m.E. einer der wenigen Fragebögen, der in 
Autoren positionieren das Konstrukt ,Angst' auf der Grundlage ihrer drei Erscheinungsformen in fremdsprachlichen Lernprozessen. Sie unterscheiden zwischen Sprechangst, Prüfungsangst und Angst vor negativer Bewertung. Sprechangst ist z.B. bei Lernenden anzutreffen, die scheu sind und jegliche Kommunikation mit anderen Menschen vermeiden oder danach streben, das begonnene Gespräch abzubrechen. Sie kann sowohl in Dyaden als auch in Kontakten mit größeren Gruppen auftreten. Die Sprachvermeidung in der Fremdsprache lässt sich in vielen Fällen auf ähnliche Strategien beim Gebrauch der Muttersprache zurückführen. ${ }^{2}$ Es ist aber auch nicht auszuschließen, dass andere Ursachen der fremdsprachlichen Angst zugrunde liegen. Die Spezifik der fremdsprachlichen Kommunikation zeichnet sich - insbesondere im Anfängerstadium - dadurch aus, dass manche (auch gesprächige) Lernende das Gelingen ihrer sprachlichen Produktionen vom wahrgenommenen und verständlichen Input im Sinne Krashens abhängig machen. Erst eine verstandene sprachliche Struktur gilt für sie als produktiv. Sie bevorzugen daher, eher den anderen zuzuhören, als selbst zu sprechen. Mit dem institutionellen Lernen einer Fremdsprache ist weiter auch die Leistungskontrolle verbunden. Sie kann Ursache für Prüfungsangst werden. ${ }^{3}$ Untersuchungen zeigen, dass es unterschiedliche Gründe für Prüfungsangst gibt. Einerseits sucht man sie in unzulänglichen Lernstilen, die eine effiziente Speicherung des Lernmaterials behindern (AIDA 1994:157). Andererseits vermögen die Lernenden ihre Leistungen nicht selten nur durch das Prisma der erreichten Testergebnisse einzuschätzen, so dass sie stark von den Prüfungsumständen (Personen, Situation, Form) abhängig werden und diese die Prüfungsangst auslösen können. Die Aufmerksamkeit wird dann eher den potentiellen Stressfaktoren und nicht dem zu prüfenden Lernmaterial geschenkt. Eine mündliche Prüfungsform löst zugleich Sprechangst aus. YoUNG (1991:429) bemerkt außerdem, dass die Testmethoden selbst zum angstauslösenden Faktor werden können. Dies

unterschiedlichen Lernerkontexten auf seine Validität und Reliabilität überprüft worden sind.

2 Die Sprechangst wird oft auf den breitangelegten Begriff der sozialen Angst bezogen. In diesem Sinne wird auch eines der Erwerbsprinzipien bei Krashen - die Herstellung eines positiven affektiven Filters - erläutert. Krashen meint, es müsse auch eine soziale Sicherheitsbasis für den Lerner (z. B. die Akzeptanz der Gruppe) hergestellt werden. (Zit. nach YounG 1991:427f.)

3 Die Prüfungsangst wird als Begriff Sensu largo eingeführt, der verschiedene Formen der Angst vor Kontrolle (etwa vor Tests, Abfragen, Zwischen- und Abschlussprüfungen) umfasst. 
geschieht z. B., wenn Lehrende den Unterricht durchaus kommunikativ gestalten und durchführen können, dann aber nur grammatische Kenntnisse überprüfen. Die dritte Art fremdsprachlicher Angst - Angst vor negativer Bewertung entsteht dann, wenn die Lernenden negative fremde Evaluation fürchten. Sie weist einige Ähnlichkeiten mit der Prüfungsangst auf, wobei die letztere sich eher nur auf eine konkrete Prüfungssituation beschränkt, die Angst vor negativer Bewertung dagegen auch unter anderen Bedingungen entstehen kann. In der Psychologie wird behauptet, dass Menschen mit größerer Feldabhängigkeit ihr Selbstbild durch fremde Eindrücke konstruieren und Situationen vermeiden, in denen dieses negativ beurteilt werden könnte (AIDA 1994:157). So weigert sich der Lernende beispielsweise, den Lehrer/die Lehrerin um eine Erklärung zu bitten, wenn er etwas nicht versteht. Wie zu sehen ist, besteht zwischen der Angst vor negativer Bewertung und der Sprechangst eine Wechselbeziehung, da die eine Art in die andere übergehen kann. HORWITZ / HORWITZ / COPE (1986:128) sehen die fremdsprachliche Angst nicht als Kombination der oben genannten Arten. Vielmehr handelte es sich um einen Komplex von Selbsteinschätzungen, Überzeugungen (sog. , learner beliefs'), Gefühlen und Verhaltensgewohnheiten. Im Kontrast zur muttersprachlichen Kommunikation wird der Lernende vor die Tatsache gestellt, dass seine fremdsprachliche Kompetenz eingeschränkt ist. Es ist wichtig $\mathrm{zu}$ betonen, dass dieser ständige, nicht selten unbewusste Vergleich zwischen dem, was in der Muttersprache und als Lernender in der Fremdsprache sprachlich erbracht werden kann, von Grund auf irreführend ist. Dies zeigt sich z.B. in der Überzeugung des Lernenden, dass nur durch formale Korrektheit die Kommunikation gelingen könne. Diese sollte man natürlich auch im Fremdsprachenunterricht anstreben, aber nicht selten verstellt sie den individuellen Charakter der sprachlichen Kompetenz und deren Entwicklung, die sich in der Muttersprache eher auf das zu erreichende kommunikative Ziel ausrichtet (abgesehen davon, dass hier und da formale Fehler auftreten; WILCZYŃSKA 1999). Die Lernenden können z. B. unterschiedliche Prioritäten setzen, die nach ihrer Überzeugung das Erlernen einer Fremdsprache garantierten: Ausspracheübungen sollten zum akzentfreien Sprechen führen, grammatische Übungen sollten für Korrektheit sorgen, zwei Jahre intensives Lernen seien genug, um eine Sprache zu lernen (Young 1991:428). ${ }^{4}$ So kann eine solche Lernereinstellung, vom Lehrer verstärkt, den Fremdsprachenunterricht und die fremdsprachliche Kommunikation zum potentiellen Ort der Angst

$4 \quad$ Vgl. die Ergebnisse von ,BALLI‘ (,Beliefs About Language Learning Inventory“) in HORWITZ (1988). 
machen. ORTEGa CEBRERos (2003) stellt sogar die Behauptung auf, der fremdsprachliche Klassenraum sei im Verhältnis zu anderen Fächern eher angstauslösenden Faktoren ausgesetzt. Solche angstauslösenden Situationen können die Lehrenden selbst schaffen, wenn sie in ihren Überzeugungen nur sich selbst die Rolle der den Unterricht steuernden Größe zuschreiben (z.B. der Lehrer/die Lehrerin muss auf alles aufpassen, um Fehler der Schüler zu vermeiden - keine Gruppen- oder Partnerarbeit, nur Frontalunterricht). Daraus resultiert auch der Charakter der Lehrenden-Lernenden-Interaktionen im Unterricht und deren asymmetrische Spezifik - die Lernenden konzentrieren sich eher auf die formale Korrektheit als auf die Realisierung der kommunikativen Absicht, die letzten Endes nicht erreicht wird. ${ }^{5}$ Dazu zählen auch die Abfrage-Prozeduren, durch die die Lernenden vor der Klasse ihre sprachlichen Leistungen beweisen sollen. YouNG (1991) zieht daraus die weitgehende Schlussfolgerung, dass nicht selten gerade das methodische Vorgehen der Lehrenden im Fremdsprachenunterricht für die Entstehung von Angstgefühlen verantwortlich gemacht werden kann. Viele Untersuchungen ergeben außerdem, dass die Einstellung der Lernenden zur eigenen Kompetenz eine Rolle spielt. So unterschätzen schüchterne Lernende ihre Kompetenz; sie kann sogar durch Selbsteinschätzungen beeinflusst werden (MACINTYRE / NOELS / ClÉMENT 1997). ${ }^{6}$ GREGERSEN / HORWITZ (2002) vergleichen ängstliche Lernende mit Perfektionisten, weil sie z.B. selten mit ihren mündlichen Sprachproduktionen zufrieden sind. Sie reagieren emotional anders auf Fehler als ihre nichtängstlichen Kommilitonen und Kommilitoninnen. BAILEY (1983) stellte nach der Analyse von Lerntagebüchern fest, dass die Angst der Lernenden ansteigt, wenn sie ihre Kompetenzmöglichkeiten mit denen der anderen Klassenkameraden/-kameradinnen vergleichen. Je mehr sich die Einschätzung eigener Kompetenz im Laufe der Zeit erhöht, desto seltener spüren die Lernenden Angstgefühle. ${ }^{7}$ So kann auch ein falscher Konkurrenzgeist zur Entstehung von Angst beitragen. STROUd / WEE (2006:301) bringen das Problem der

5 Andererseits muss jedoch vermerkt werden, dass die Lernenden Korrekturmaßnahmen von ihren Lehrern und Lehrerinnen erwarten.

6 Es ist außerdem zu bemerken, dass die Sprechangst im Allgemeinen mehr mit subjektiver als mit objektiver Kompetenzeinschätzung korreliert. Wie schon erwähnt, unterschätzen ängstliche Lerner ihre Kompetenz, nichtängstliche Lerner tendieren zur Überschätzung der eigenen Kompetenz.

7 ATAY / KURT (2006:111) machen darauf aufmerksam, dass eine hohe Sprachkompetenz nicht unbedingt geringe Angstgefühle im Fremdsprachenunterricht bedeuten muss. 
Identitätsangst zur Sprache. Sie sind der Meinung, dass nicht selten Mitlernende aufeinander negativen Einfluss haben, so dass manche Lernende z.B. Vergewisserungsfragen vermeiden, um nicht als ,attention-seeker' im Klassenverband zu gelten. Als wichtig ist im Kontext der angstauslösenden Faktoren festzuhalten, dass die fremdsprachliche Angst sich kontinuierlich als Folge früherer negativer Erfahrungen mit dem Lernen einer Fremdsprache entwickelt (MACINTYRE / GARDNER 1991, AIDA 1994). MACINTYRE / GARDNER (1994, zit. nach ElLIS 2004) weisen darauf hin, dass fremdsprachliche Angst sich auf drei Prozessebenen während des Sprachenlernens entwikkeln kann. Zum einen ist da die Input-Ebene, auf der die Lernenden das neue Material verstehen und verarbeiten sollen. Zum anderen haben wir die Speicherungsebene, auf der das Neue organisiert wird. Schließlich gibt es die Output-Ebene, auf der das Gelernte in Form von Sprachfertigkeiten und -kenntnissen gebraucht werden soll. Vor diesem Hintergrund behaupten BAiley / OwnUegBuZIE / DAley (2000), dass Personen mit höchsten Angstwerten auf diesen Stufen eher älter sind und bescheidenere Erwartungen an den Unterricht haben. Sie haben auch ein geringeres Selbstwertgefühl und eine niedrigere Ausbildung. Wie schon angeführt, werden die negativen Einflüsse z.B. mit Performanzergebnissen der Probanden in Zusammenhang gebracht. So stellt sich heraus, dass Angstgefühle mit Sprech- und Schreibaufgaben oder aber mit allgemeinen Kompetenztests negativ korrelieren können, obwohl nicht immer alle Fertigkeiten genauso negativ beeinflusst werden (YounG 1991). SAITO / HORWITZ / GARZA (1999) fanden heraus, dass die Leseangst eher als Folge aufgabenspezifischer Schwierigkeiten und nicht als Ursache der Leseprobleme betrachtet werden sollte. Negative Wirkung kann die Angst auch auf unterschiedliche Selbstkonzepte wie etwa Selbstwertgefühl, Selbstvertrauen oder Selbstwirksamkeit haben. ${ }^{8}$ PHILLIPS (1992) hat beobachtet, dass die Lernenden davon überzeugt sind, ihre Ängstlichkeit hätte einen entscheidenden negativen Einfluss auf ihre mündlichen Produktionen, obwohl statistisch gesehen keine Korrelation bestand. ATAY / KURT (2006) weisen auch darauf hin, dass z. B. Lehrer/innen, die Angst vor schriftlichen Aufgaben haben, diese auch ihren Schülern und Schülerinnen vorenthalten. SPARKS / GANSCHOW (1995), GANSCHOW / SPARKS (1996) behaupten im Rahmen der von ihnen aufgestellten Hypothese über ,Linguistic Coding Differences', mangelnde Fähigkeiten und Fertigkeiten in L1 könnten negative Auswirkungen auf Input- und Output-Prozesse in L2 haben, so dass sie auch zur

8 Vgl. MissLER (1999:178-180). CHAO (2004) hat gezeigt, dass es einen signifikanten Zusammenhang zwischen Angstgefühlen und emotionaler Intelligenz gibt. 
Angstauslösung oder -steigerung führen würden. ${ }^{9}$ DJIGUNOVIC (2006) kommt zu der Erkenntnis, dass ängstlichere Lernende längere Texte in L2 als in L1 herstellen, wobei kontinuierliche Redeansätze in L1 und L2 kürzer als bei weniger ängstlichen Lernenden sind. Sie machen längere Satzpausen, weniger Wiederholungen und mehr Fehlstarts. Andere interessante Ergebnisse liefert die Studie von GREGERSEN (2005), in der einige nonverbale Verhaltensweisen von ängstlichen Lernenden beschrieben werden. So sind solche Lerner/innen weniger aktiv in ihrem Gesichtsausdruck, haben weniger Augenkontakte mit den Lehrenden und machen nicht so oft von Gesten Gebrauch. Einige Untersuchungen haben ergeben, dass es auch positive Folgen von Angstgefühlen geben kann. So wird von HORWITZ (1990, zit. nach OXFORD 1999:61) behauptet, Angst könne bei der Lösung sehr einfacher Aufgaben förderlich sein. Die Breite unterschiedlicher, manchmal entgegengesetzter Forschungsergebnisse geht darauf zurück, dass Angst als wissenschaftliches Problem nicht selten ein verschwommenes Konstrukt darstellt. Es werden z.B. verschiedene Definitionen von Angst herangezogen und diverse Messinstrumente angewendet (vgl. YounG 1991, GROTJAHN 2004).

\section{Empirische Untersuchung - Angstgefühle von Studienanfängern in der Germanistik}

Im folgenden Kapitel werden die Ergebnisse einer empirischen Untersuchung dargestellt, die unter Studienanfängern in der Germanistik durchgeführt wurde. Ziel dieser Studie war es zu erfahren, ob und in welchem Grade die Studienanfänger/innen ihre eventuellen fremdsprachlichen Angstgefühle nachvollziehen und einschätzen können. Im Fokus stehen die Schulerfahrungen der Lernenden. Da zwei verschiedene Studiengänge - Studierende des Magisterstudiums (5 Jahre) und Studierende des Berufsstudiums (3 Jahre) - ausgewählt wurden, sollte außerdem untersucht werden, ob es zwischen diesen Gruppen signifikante Unterschiede gibt. Darüber hinaus interessiert, welche Arten von Angst, bezogen auf die bisher in der Literatur vorgeschlagenen Differenzierungen, vorkommen und wie stark sie bei den Probandengruppen zu diagnostizieren sind. Neben empirischen Befunden erscheinen die daraus resultierenden didaktischen Schlussfolgerungen für die Gestaltung von universitären Sprachlehr- und -lernprozessen von besonderer Relevanz.

9 HoRwiTZ (2000:257) widerlegt diese Argumente, weil sie viele Fälle von ängstlichen Studierenden beobachten konnte, die keine kognitiven Defizite in ihrer L1 aufwiesen und außerdem zu den guten Studierenden gehörten. 
Angstgefühle und deren mögliche Auswirkungen

\section{Versuchspersonen}

Die erste Gruppe bildeten 51 Studierende (40 Frauen, 11 Männer) des fünfjährigen Magisterstudiums am Institut für Germanistik in Szczecin. Die zweite Gruppe bestand aus 46 Studierenden (38 Frauen, 8 Männer) des dreijährigen Berufsstudiums. Diese studieren an zwei Institutionen: dem Institut für Germanistik an der Technischen Universität Koszalin (34 Studierende) und dem Institut für Fremdsprachen am Collegium Balticum in Szczecin (12 Studierende).

\section{Erhebungsinstrument und Durchführung}

Zur Durchführung der Untersuchung wurde der standardisierte Test „FLCAS“ („Foreign Language Classroom Anxiety Scale“) von Horwitz verwendet. Die Befragung erfolgte Ende November 2006. Wie oben dargestellt, besteht „FLCAS“ aus 33 Items, denen fünf mögliche Antworten auf einer 5-PunkteLikert-Skala (1: starke Ablehnung der Aussage; 2: Ablehnung der Aussage; 3: schwer präzisierbare oder keine Antwort; 4: Zustimmung zur Aussage; 5: starke Zustimmung zur Aussage) zugeordnet sind. Der Fragebogen wurde aus dem Englischen ins Polnische übersetzt und berücksichtigte auch die Schulerfahrungen der Probanden/innen. In der Instruktion zum Fragebogen wurde das zu untersuchende Problem nicht direkt erwähnt, sondern um Antworten zu fremdsprachlichen Schulerfahrungen gebeten. Zur Feststellung der Genauigkeit der erhobenen Werte (Reliabilität) wurde ,Cronbachs Alpha“ berechnet. Bei der ersten Gruppe betrug der Wert $\alpha=.92$, bei der zweiten Gruppe $\alpha=.94 .^{10}$ Diese Ergebnisse zeugen von großer innerer Konstruktkonsistenz des Fragebogens. Da die Antworten auf der 5-Punkte-LikertSkala bestimmt wurden, betrug der höchste Angstgrad 165 Punkte (33 Items mal 5). ${ }^{11}$ Zusätzlich wurden die zu Untersuchenden gefragt, wann sie ihren schulischen Deutschunterricht begonnen haben - in der Grundschule (Alter 713), im Gymnasium (Alter 13-16) oder im Lyzeum (Alter 16-19).

10 Zum Vergleich bei HorwitZ et alii (1991) $-\alpha=.93$, bei AIDA (1994) $-\alpha=.94$.

11 Bei den Items 2, 5, 8, 11, 14, 18, 22, 28, 32 wurden die Punkte umgekehrt berechnet. 
Krzysztof Nerlicki

\section{Ergebnisse}

Zuerst sind die Mittelwerte der erreichten Punkte von 165 möglichen und die Standardabweichungen bei den untersuchten Gruppen berechnet worden.

\begin{tabular}{|c|c|c|}
\hline Gruppe & Mittelwert & Standardabweichung \\
\hline Magisterstudium & 79,9 & 22,9 \\
\hline Berufsstudium & 88,6 & 26,3 \\
\hline
\end{tabular}

Aus der Tabelle folgt, dass die meisten Werte um die Hälfte der möglichen Punktezahl lagen. Die Werte rangieren zwischen 44 und 126 (Magisterstudium) sowie 45 und 144 (Berufsstudium). Die Mittelwerte sind um einige Punkte höher als bei der Gruppe der Berufsstudierenden, aber der zweiseitige t-Test hat keine signifikanten Unterschiede zwischen den Gruppen ergeben $\mathrm{t}(90)=1,72 ; \mathrm{p}<0,05$. Deswegen kann nicht die Schlussfolgerung gezogen werden, dass die Gruppe der Berufsstudierenden ängstlicher ist als die der Magisterstudierenden. Geschlechtsspezifisch verteilen sich die Mittelwerte wie folgt:

\begin{tabular}{|c|c|}
\hline Magisterstudium & Mittelwert \\
\hline Frauen $(\mathrm{N}=40)$ & 82,9 \\
\hline Männer $(\mathrm{N}=11)$ & 69,0 \\
\hline
\end{tabular}

\begin{tabular}{|l|c|}
\hline Berufsstudium & Mittelwert \\
\hline Frauen (N=38) & 92,1 \\
\hline Männer (N=8) & 71,7 \\
\hline
\end{tabular}

Da die Gruppe der männlichen Probanden unterrepräsentiert ist, ist auf die Berechnung der Unterschiedssignifikanz verzichtet worden, obwohl die Werte zeigen, dass Frauen ängstlicher als Männer sein können oder aber, was auch die psychologische Forschung zugesteht, Männer nur ungern ihre eventuellen Angstgefühle eingestehen wollen. Wenn die Mittelwerte der Gesamtprobe nach der Länge des Deutschunterrichts angeordnet werden, sind folgende Ergebnisse zu verzeichnen:

\begin{tabular}{|c|c|}
\hline Lernen Deutsch seit & Mittelwert \\
\hline der Grundschule N=53 & 80,9 \\
Magisterstudium - 29; Berufsstudium - 24 & (Magisterstudium - 79,7; Berufsstudium - 82,4) \\
\hline
\end{tabular}


Angstgefühle und deren mögliche Auswirkungen

\begin{tabular}{|c|c|}
\hline dem Gymnasium N=25 & 83,2 \\
Magisterstudium - 13; Berufsstudium - 12 & (Magisterstudium 72,9; Berufsstudium 94,4) \\
\hline dem Lyzeum N=15 & 92,4 \\
Magisterstudium - 6; Berufsstudium - 9 & (Magisterstudium 94; Berufsstudium 91,3) \\
\hline
\end{tabular}

Die zahlreichste Gruppe bilden die Studierenden, die ihren Deutschunterricht in der Grundschule begannen - insgesamt 53 Personen. Der Mittelwert beträgt bei dieser Gruppe 80,9 Punkte. Bei der nächsten Gruppe, die erst im Gymnasium mit dem Deutschunterricht angefangen hat, liegt der Wert ein wenig höher $(83,2)$, die Teilwerte im Magisterstudium und Berufstudium unterscheiden sich indes deutlich (72,9 und 94,4). Die Gruppe derjenigen, die Deutsch erst auf dem Lyzeum lernten, zeigt den höchsten Mittelwert - 92,4. Um zu überprüfen, ob die Unterschiede zwischen den genannten Gruppen statistisch signifikant sind, wurde eine ,ANOVA' durchgeführt. Die Unterschiede sind nicht signifikant $-\mathrm{F}(3,09)=1,27, \mathrm{p}=0,28$. Bei der Interpretation der Daten wurden die Items den von ORTEGA CEBREROS (2003) festgestellten Faktoren zugeordnet. Die Autorin hat die von HORWITZ / HORWITZ / COPE (1986) vorgeschlagenen drei Faktoren, d.h. Sprechangst, Prüfungsangst und Angst vor negativer Bewertung, weiter präzisiert. Bei ihr finden sich Faktoren wie Sprechangst, Verstehensangst, Prüfungsangst und generelle Angst, die innerhalb und außerhalb des Klassenraumes zu positionieren sind. Auf dieser Basis können die Items den folgenden Arten von Angst zugeordnet werden:

\begin{tabular}{|l|l|}
\hline \multicolumn{1}{|c|}{ Arten von Angst } & \multicolumn{1}{c|}{ Items } \\
\hline Sprechangst & $\begin{array}{l}1,2,3,7,9,13,14,18,19,20,23,24,27,30,31,32, \\
33\end{array}$ \\
\hline Verstehensangst & $4,15,29$ \\
\hline Prüfungsangst & $8,10,21$ \\
\hline $\begin{array}{l}\text { Angstfaktoren, die innerhalb und au- } \\
\text { Berhalb des Klassenraumes entstehen }\end{array}$ & $5,6,11,12,16,17,22,25,26,28$ \\
\hline
\end{tabular}

Im Folgenden werden die Daten zu den jeweiligen Angstfaktoren angegeben und besprochen. Da die Analyse generell keine signifikanten Unterschiede zwischen den Gruppen ergibt, werden nur diejenigen Punkte separat kommentiert, bei denen die erreichten prozentualen Werte in den Gruppen deutlich voneinander abweichen.

12 Die Gesamtzahl der Probanden/innen betrug 97 Personen, 4 davon haben aber keine Angabe zu diesem Punkt gemacht. 
Krzysztof Nerlicki

\section{Sprechangst}

Zuerst werden die Ergebnisse zum Faktor Sprechangst diskutiert. Diesem Faktor werden bei ORTEGA CEBREROS (2003) 17 Items zugeordnet. $^{13}$

Item 1: Beim Deutschsprechen in der Klasse habe ich mich nie ganz sicher gefühlt. ${ }^{14}$

\begin{tabular}{|c|c|c|c|c|c|c|}
\hline Gruppe & $\begin{array}{c}\text { starke Ab- } \\
\text { lehnung }\end{array}$ & Ablehnung & $\begin{array}{c}\text { unent- } \\
\text { schieden }\end{array}$ & $\begin{array}{c}\text { Zustim- } \\
\text { mung }\end{array}$ & $\begin{array}{c}\text { starke Zu- } \\
\text { stimmung }\end{array}$ & $\begin{array}{c}\text { Mittelwert } \\
\text { (Standard- } \\
\text { abwei- } \\
\text { chung) }\end{array}$ \\
\hline $\begin{array}{c}\text { Magister- } \\
\text { Studium }\end{array}$ & 18 & 29 & 8 & 22 & 23 & 3,03 \\
\hline $\begin{array}{c}\text { Berufs- } \\
\text { Studium }\end{array}$ & 11 & 19 & 9 & 39 & 21 & 3,41 \\
& & & $1,48)$ \\
\hline
\end{tabular}

Unter den Berufsstudierenden geben $60 \%$ Unsicherheitsgefühle beim Sprechen zu, bei den Magisterstudierenden sind dies nur $45 \%$. Der Mittelwert der zweiten Gruppe zeigt, dass die Tendenz zur Zustimmung neigt, dass aber die Unterschiede zwischen den Gruppen statistisch nicht signifikant sind. Das Problem der Sicherheit/Unsicherheit beim Sprechen ist noch einmal mit dem Item 18 überprüft worden.

Item 18: Beim Sprechen im Deutschunterricht habe ich mich sicher gefühlt.

\begin{tabular}{|c|c|c|c|c|c|c|}
\hline Gruppe & $\begin{array}{c}\text { starke Ab- } \\
\text { lehnung }\end{array}$ & Ablehnung & $\begin{array}{c}\text { unent- } \\
\text { schieden }\end{array}$ & $\begin{array}{c}\text { Zustim- } \\
\text { mung }\end{array}$ & $\begin{array}{c}\text { starke Zu- } \\
\text { stimmung }\end{array}$ & $\begin{array}{c}\text { Mittelwert } \\
\text { (Standard- } \\
\text { abwei- } \\
\text { chung) }\end{array}$ \\
\hline $\begin{array}{c}\text { Magister- } \\
\text { Studium }\end{array}$ & 8 & 31 & 14 & 22 & 25 & $\begin{array}{c}3,25 \\
(1,35)\end{array}$ \\
\hline $\begin{array}{c}\text { Berufs- } \\
\text { Studium }\end{array}$ & 15 & 24 & 26 & 26 & 9 & $\begin{array}{c}2,89 \\
(1,21)\end{array}$ \\
\hline
\end{tabular}

Die Korrelationen innerhalb der Gruppen zwischen den beiden Items betragen im Magisterstudium $r=.76$ und im Berufsstudium $r=.64$. Der niedrigere Wert

13 Die Faktorenanalyse kann je nach angenommenen Angstfaktoren unterschiedliche Skalen ergeben. AIDA (1994) ordnet z.B. dem Faktor Sprechangst 18 Items, dem Faktor Angst vor Versagen 4 Items, dem Faktor Sprechlust mit Muttersprachlern 3 Items und dem Faktor negative Gefühle 2 Items zu. 6 Items konnten nicht zugeordnet werden.

14 Die Items wurden für die Zwecke dieses Beitrags aus dem Polnischen übersetzt. 
in der zweiten Gruppe ist auf die recht hohe Anzahl von unentschiedenen Antworten zurückzuführen. Die Annahme, wonach die Studierenden im Berufsstudium weniger sicher oder unsicherer beim Sprechen wären, kann auf dieser Datenbasis nicht gestützt werden. Die Unterschiede zwischen den Gruppen sind statistisch nicht signifikant. Angstgefühle entstehen nicht selten auch dann, wenn man die Fehler, die ohnehin beim Fremdsprachenlernen natürlich sind, allzu ernst nimmt. Item 2 bezog sich im Allgemeinen auf das Verhältnis der Lernenden zu Fehlern. Mit Item 19 sind die Probanden und Probandinnen gefragt worden, ob sie Angst davor haben, dass der Lehrende ihre Fehler korrigiert.

Item 2: Ich machte mir keine Sorgen um meine Sprechfehler im Unterricht.

\begin{tabular}{|c|c|c|c|c|c|c|}
\hline Gruppe & $\begin{array}{c}\text { starke Ab- } \\
\text { lehnung }\end{array}$ & Ablehnung & $\begin{array}{c}\text { unent- } \\
\text { schieden }\end{array}$ & $\begin{array}{c}\text { Zustim- } \\
\text { mung }\end{array}$ & $\begin{array}{c}\text { starke Zu- } \\
\text { stimmung }\end{array}$ & $\begin{array}{c}\text { Mittelwert } \\
\text { (Standard- } \\
\text { abwei- } \\
\text { chung) }\end{array}$ \\
\hline $\begin{array}{c}\text { Magister- } \\
\text { Studium }\end{array}$ & 12 & 23 & 10 & 33 & 22 & $\begin{array}{c}3,29 \\
(1,36)\end{array}$ \\
\hline $\begin{array}{c}\text { Berufs- } \\
\text { Studium }\end{array}$ & 15 & 48 & 9 & 19 & 9 & $\begin{array}{c}2,58 \\
(1,22)\end{array}$ \\
\hline
\end{tabular}

Aus der Tabelle geht hervor, dass die beim Sprechen auftauchenden Fehler weniger die Studierenden im Magisterstudium beschäftigen. Insgesamt haben $55 \%$ diese Frage positiv beantwortet. Dagegen geben $63 \%$ der Studierenden im Berufsstudium 63\% Antworten, die das Gegenteil behaupten. Diese Meinungen können daraus resultieren, dass die Berufsstudierenden im Allgemeinen eine niedrigere Sprachkompetenz haben als ihre Kommilitonen und Kommilitoninnen im Magisterstudium, so dass auch Fehler einen stärkeren emotionalen Einfluss ausüben können.

Item 19: Ich hatte Angst, dass der Lehrer jeden Fehler korrigieren wird.

\begin{tabular}{|c|c|c|c|c|c|c|}
\hline Gruppe & $\begin{array}{c}\text { starke Ab- } \\
\text { lehnung }\end{array}$ & Ablehnung & $\begin{array}{c}\text { unent- } \\
\text { schieden }\end{array}$ & $\begin{array}{c}\text { Zustim- } \\
\text { mung }\end{array}$ & $\begin{array}{c}\text { starke Zu- } \\
\text { stimmung }\end{array}$ & $\begin{array}{c}\text { Mittelwert } \\
\text { (Standard- } \\
\text { abwei- } \\
\text { chung) }\end{array}$ \\
\hline $\begin{array}{c}\text { Magister- } \\
\text { Studium }\end{array}$ & 39 & 29 & 18 & 8 & 6 & $\begin{array}{c}2,11 \\
(1,19)\end{array}$ \\
\hline $\begin{array}{c}\text { Berufs- } \\
\text { Studium }\end{array}$ & 22 & 28 & 26 & 17 & 6 & $\begin{array}{c}2,58 \\
(1,20)\end{array}$ \\
\hline
\end{tabular}


Die Daten zeigen, dass dieser Faktor in der Gruppe der Berufsstudierenden eher als potentielle Angstquelle betrachtet wird (23\%). Viele Proband(inn)en (26\%) haben keine eindeutige Entscheidung treffen können. Statistisch gesehen kann man jedoch keinen signifikanten Unterschied feststellen. Es gibt auch in den beiden Gruppen keine signifikante Korrelation zwischen den Items 2 und 19. Angstgefühle können auch dann entstehen, wenn man das Sprechen in einer Fremdsprache vor allem von der Regelmenge und ihrer Kenntnis abhängig macht.

Item 30: Mich bedrückte eine Menge von Regeln, die gelernt werden mussten, um Deutsch sprechen zu können.

\begin{tabular}{|c|c|c|c|c|c|c|}
\hline Gruppe & $\begin{array}{c}\text { starke Ab- } \\
\text { lehnung }\end{array}$ & Ablehnung & $\begin{array}{c}\text { unent- } \\
\text { schieden }\end{array}$ & $\begin{array}{c}\text { Zustim- } \\
\text { mung }\end{array}$ & $\begin{array}{c}\text { Starke Zu- } \\
\text { stimmung }\end{array}$ & $\begin{array}{c}\text { Mittelwert } \\
\text { (Standard- } \\
\text { abweichung) }\end{array}$ \\
\hline $\begin{array}{c}\text { Magister- } \\
\text { Studium }\end{array}$ & 25 & 39 & 11 & 21 & 1 & $\begin{array}{c}2,35 \\
(1,14)\end{array}$ \\
\hline $\begin{array}{c}\text { Berufs- } \\
\text { Studium }\end{array}$ & 13 & 33 & 20 & 13 & 20 & $\begin{array}{c}2,93 \\
(1,35)\end{array}$ \\
\hline
\end{tabular}

Wenn man die Prozentwerte in den beiden Gruppen vergleicht, sieht man, dass die Studierenden im Berufsstudium die Sprechfähigkeit eher von Regelkenntnissen abhängig machen als die Studierenden im Magisterstudium. Die Unterschiede sind statistisch signifikant $\left(\chi^{2}=11,28\right.$, df $\left.=4, p<0,05\right)$. Überprüft wurde auch, ob die Ergebnisse der Items 2 und 30 innerhalb der Gruppen miteinander korrelieren. In der ersten Gruppe kann eine schwache negative Korrelation $(r=-.41)$ festgestellt werden. In der zweiten Gruppe gibt es keine Korrelation zwischen diesen Items $(r=.08)$. Die Items 3 und 20 beziehen sich auf einen Faktor, der sehr oft zu Angst im Unterricht führt, und zwar auf das Abfragen.

Item 3: Ich zitterte, als ich wusste, ich werde im Unterricht aufgerufen.

\begin{tabular}{|c|c|c|c|c|c|c|}
\hline Gruppe & $\begin{array}{c}\text { starke Ab- } \\
\text { lehnung }\end{array}$ & Ablehnung & $\begin{array}{c}\text { unent- } \\
\text { schieden }\end{array}$ & $\begin{array}{c}\text { Zustim- } \\
\text { mung }\end{array}$ & $\begin{array}{c}\text { Starke Zu- } \\
\text { stimmung }\end{array}$ & $\begin{array}{c}\text { Mittelwert } \\
\text { (Standard- } \\
\text { abwei- } \\
\text { chung) }\end{array}$ \\
\hline $\begin{array}{c}\text { Magister- } \\
\text { Studium }\end{array}$ & 31 & 37 & 8 & 20 & 4 & $\begin{array}{c}2,27 \\
(1,21)\end{array}$ \\
\hline $\begin{array}{c}\text { Berufs- } \\
\text { Studium }\end{array}$ & 24 & 35 & 4 & 17 & 20 & $\begin{array}{c}2,73 \\
(1,49)\end{array}$ \\
\hline
\end{tabular}


In beiden Gruppen überwiegen negative Antworten auf diese Frage (68\% und $59 \%$ ). Unter den Berufsstudierenden ist die Anzahl derjenigen, die dieser Meinung zustimmen, um $13 \%$ höher als bei den Magisterstudierenden. Bei der statistischen Berechnung $\left(\chi^{2}=11,84, \mathrm{df}=4, \mathrm{p}<0,05\right)$ ist festzustellen, dass die Unterschiede zwischen den Gruppen signifikant sind. In diesen Zusammenhang gehört auch Item 20.

Item 20: Ich spürte mein Herz schlagen, immer wenn ich im Unterricht abgefragt wurde.

\begin{tabular}{|c|c|c|c|c|c|c|}
\hline Gruppe & $\begin{array}{c}\text { starke Ab- } \\
\text { lehnung }\end{array}$ & Ablehnung & $\begin{array}{c}\text { unent- } \\
\text { schieden }\end{array}$ & $\begin{array}{c}\text { Zustim- } \\
\text { mung }\end{array}$ & $\begin{array}{c}\text { Starke Zu- } \\
\text { stimmung }\end{array}$ & $\begin{array}{c}\text { Mittelwert } \\
\text { (Standard- } \\
\text { abwei- } \\
\text { chung) }\end{array}$ \\
\hline $\begin{array}{c}\text { Magister- } \\
\text { Studium }\end{array}$ & 31 & 20 & 12 & 21 & 16 & $\begin{array}{c}2,70 \\
(1,50)\end{array}$ \\
\hline $\begin{array}{c}\text { Berufs- } \\
\text { Studium }\end{array}$ & 24 & 24 & 11 & 28 & 13 & $\begin{array}{c}2,82 \\
(1,41)\end{array}$ \\
\hline
\end{tabular}

Die Tatsache, im Unterricht abgefragt zu werden, hat in beiden Gruppen prozentuell gesehen ähnliche Ergebnisse gezeigt. Wenn man die Korrelationen zwischen den Items 3 und 20 misst, ist zu erkennen, dass sie in der Magistergruppe $r=.54$ und in der Berufsgruppe $r=.69$ betragen. Daraus kann man schließen, dass es in der Berufsgruppe mehr Studierende gab, die sowohl vor dem ihnen bekannten Zeitpunkt des Abfragens wie auch direkt während dieser Zeit in Angst gerieten. Ein ähnliches Problem wird mit den Items 33 und 9 überprüft. Hier geht es darum, ob die Lernenden Angst spüren, wenn sie Fragen gestellt bekommen, auf die sie sich früher nicht vorbereiten konnten und ob sie in Panik gerieten, wenn sie zum Sprechen ohne frühere Vorbereitung aufgefordert wurden.

Item 33: Ich war aufgeregt, als der Lehrer mir Fragen stellte, auf die ich früher keine Antwort hatte vorbereiten können.

\begin{tabular}{|c|c|c|c|c|c|c|}
\hline Gruppe & $\begin{array}{c}\text { starke Ab- } \\
\text { lehnung }\end{array}$ & Ablehnung & $\begin{array}{c}\text { unent- } \\
\text { schieden }\end{array}$ & $\begin{array}{c}\text { Zustim- } \\
\text { mung }\end{array}$ & $\begin{array}{c}\text { Starke Zu- } \\
\text { stimmung }\end{array}$ & $\begin{array}{c}\text { Mittelwert } \\
\text { (Standard- } \\
\text { abweichung) }\end{array}$ \\
\hline $\begin{array}{c}\text { Magister- } \\
\text { Studium }\end{array}$ & 27 & 29 & 14 & 22 & 8 & $\begin{array}{c}2,52 \\
(1,31)\end{array}$ \\
\hline $\begin{array}{c}\text { Berufs- } \\
\text { Studium }\end{array}$ & 13 & 33 & 26 & 13 & 15 & $\begin{array}{c}2,84 \\
(1,26)\end{array}$ \\
\hline
\end{tabular}


Die Prozentwerte verteilen sich fast gleich, wobei die Anzahl der stark Zustimmenden im Falle der Berufsstudierenden doppelt so hoch ist wie bei den Magisterstudierenden. Außerdem ist die Anzahl der Unentschiedenen bei den Berufsstudierenden auch zweimal höher. Die Verteilung der zustimmenden Antworten erlaubt die vorsichtige Schlussfolgerung, dass sich in dieser Gruppe die Tendenz abzeichnet, doch Angstgefühle bei unerwarteten Fragen zu spüren.

Item 9: Ich geriet in Panik, wenn ich ohne frühere Vorbereitung sprechen musste.

\begin{tabular}{|c|c|c|c|c|c|c|}
\hline Gruppe & $\begin{array}{c}\text { starke Ab- } \\
\text { lehnung }\end{array}$ & Ablehnung & $\begin{array}{c}\text { unent- } \\
\text { schieden }\end{array}$ & $\begin{array}{c}\text { Zustim- } \\
\text { mung }\end{array}$ & $\begin{array}{c}\text { Starke Zu- } \\
\text { stimmung }\end{array}$ & $\begin{array}{c}\text { Mittelwert } \\
\text { (Standard- } \\
\text { abweichung) }\end{array}$ \\
\hline $\begin{array}{c}\text { Magister- } \\
\text { Studium }\end{array}$ & 31 & 35 & 8 & 12 & 14 & $\begin{array}{c}2,41 \\
(1,40)\end{array}$ \\
\hline $\begin{array}{c}\text { Berufs- } \\
\text { Studium }\end{array}$ & 15 & 39 & 9 & 26 & 11 & $\begin{array}{c}2,78 \\
(1,29)\end{array}$ \\
\hline
\end{tabular}

Auch in diesem Punkt haben die meisten Probanden/innen der genannten Meinung negativ gegenübergestanden, wobei die Anzahl der positiven Antworten bei den Berufsstudierenden um $11 \%$ höher lag. Es wurde überprüft, ob es eine Korrelation zwischen den Werten in Item 3 (Ich zitterte, als ich wusste, ich werde im Unterricht abgefragt) und diesem Item gab. Interessanterweise betrug diese Korrelation in der ersten Gruppe nur $\mathrm{r}=.33$ und in der zweiten Gruppe $r=.75$. Das bedeutet, dass diese Angstgefühle häufiger zusammen bei der Gruppe der Berufsstudierenden vorkommen. Es gibt keinen signifikanten Unterschied zwischen den Gruppen. Die Korrelationen zwischen den Items 33 und 9 erreichen in beiden Gruppen einen mittleren Wert $(r=.58 ; r=.64)$. Mit den Items 27 und 24 sollte ermittelt werden, inwieweit die unterrichtliche Sprechkommunikation zu Ängsten führen kann. Es ging darum, wie die Lernenden im Allgemeinen ihre Sprechbeiträge einschätzen, wobei das Item 24 eher das Individuelle betonte, und die Lernenden danach gefragt wurden, ob sie sich sicher fühlen, wenn sie vor die Klasse treten und reden.

Item 27: Ich fühlte mich aufgeregt und verwirrt, wenn ich im Unterricht redete.

\begin{tabular}{|c|c|c|c|c|c|c|}
\hline Gruppe & $\begin{array}{c}\text { starke Ab- } \\
\text { lehnung }\end{array}$ & Ablehnung & $\begin{array}{c}\text { unent- } \\
\text { schieden }\end{array}$ & $\begin{array}{c}\text { Zustim- } \\
\text { mung }\end{array}$ & $\begin{array}{c}\text { Starke Zu- } \\
\text { stimmung }\end{array}$ & $\begin{array}{c}\text { Mittelwert } \\
\text { (Standard- } \\
\text { abweichung) }\end{array}$ \\
\hline $\begin{array}{c}\text { Magister- } \\
\text { Studium }\end{array}$ & 36 & 36 & 6 & 16 & 6 & $\begin{array}{c}2,20 \\
(1,26)\end{array}$ \\
\hline $\begin{array}{c}\text { Berufs- } \\
\text { Studium }\end{array}$ & 33 & 19 & 19 & 19 & 9 & $\begin{array}{c}2,52 \\
(1,36)\end{array}$ \\
\hline
\end{tabular}


Angstgefühle und deren mögliche Auswirkungen

Item 24: Ich fühlte mich unsicher, wenn ich vor die ganze Klasse treten und sprechen sollte.

\begin{tabular}{|c|c|c|c|c|c|c|}
\hline Gruppe & $\begin{array}{c}\text { starke Ab- } \\
\text { lehnung }\end{array}$ & Ablehnung & $\begin{array}{c}\text { unent- } \\
\text { schieden }\end{array}$ & $\begin{array}{c}\text { Zustim- } \\
\text { mung }\end{array}$ & $\begin{array}{c}\text { Starke Zu- } \\
\text { stimmung }\end{array}$ & $\begin{array}{c}\text { Mittelwert } \\
\text { (Standard- } \\
\text { abwei- } \\
\text { chung) }\end{array}$ \\
\hline $\begin{array}{c}\text { Magister- } \\
\text { Studium }\end{array}$ & 20 & 23 & 16 & 23 & 18 & $\begin{array}{c}2,96 \\
(1,41)\end{array}$ \\
\hline $\begin{array}{c}\text { Berufs- } \\
\text { Studium }\end{array}$ & 15 & 28 & 11 & 22 & 24 & $\begin{array}{c}3,10 \\
1,44\end{array}$ \\
\hline
\end{tabular}

Betrachtet man die Mittelwerte in den beiden Tabellen, so kommt man zu dem Schluss, dass die unterrichtlichen Situationen, in denen die Studierenden vor die Klasse treten und sprechen sollten, für etwa die Hälfte von ihnen nicht komfortabel waren. Dies ist eine ganz normale Reaktion, wenn man sich dessen bewusst wird, dass die schulischen mündlichen Beiträge der Lernenden auch mit einer Benotung verbunden waren. Die Ergebnisse zwischen den Items 24 und 27 korrelieren zudem hoch - in der ersten Gruppe $r=.69$, in der zweiten $r=.72$, was zu der Annahme führt, dass die meisten Meinungen bei diesen zwei Items nicht auseinandergehen. Die möglichen Angstgefühle beim Reden wurden also mit dem gesamten Unterricht assoziiert und umso mehr mit den eigenen Auftritten vor der Klasse. Die nächsten drei Items (7, 23, 31) beziehen sich auf Angstgefühle, die im Kontext der Lernergruppe entstehen können. Es ist nämlich nicht selten so, dass einige Schüler/innen ihre eigenen fremdsprachlichen Fähigkeiten und Fertigkeiten durch das Prisma der ständigen Vergleiche mit den Klassenkameraden/innen einzuschätzen versuchen. In diesen Fällen kommt es nicht selten zur Unterschätzung eigener Lernpotentiale und -leistungen, so dass diese Vergleiche auch Angstgefühle verursachen. Andererseits liegt es an der Lerngruppe selbst (z.B. am Grad der Beziehungen zwischen den Lernenden), ob ein eher für fremde Urteile anfälliger Lernender den negativen Reaktionen des Lernumfeldes standhält oder nicht.

Item 7: Ich dachte immer daran, dass andere Schüler besser sind als ich.

\begin{tabular}{|c|c|c|c|c|c|c|}
\hline Gruppe & $\begin{array}{c}\text { starke Ab- } \\
\text { lehnung }\end{array}$ & Ablehnung & $\begin{array}{c}\text { unent- } \\
\text { schieden }\end{array}$ & $\begin{array}{c}\text { Zustim- } \\
\text { mung }\end{array}$ & $\begin{array}{c}\text { starke Zu- } \\
\text { stimmung }\end{array}$ & $\begin{array}{c}\text { Mittelwert } \\
\text { (Standard- } \\
\text { abweichung) }\end{array}$ \\
\hline $\begin{array}{c}\text { Magister- } \\
\text { Studium }\end{array}$ & 33 & 27 & 4 & 24 & 12 & 2,52 \\
& & 26 & 19 & 19 & 15 & 2,84 \\
\hline $\begin{array}{c}\text { Berufs- } \\
\text { Studium }\end{array}$ & 19 & & & & $(1,36)$ \\
\hline
\end{tabular}


Die Mehrheit der Antworten ist in beiden Gruppen negativ (60 \%, $45 \%$ ). $36 \%$ (Magisterstudierende) und $34 \%$ (Berufsstudierende) vertreten die Meinung, dass sie ständig daran denken, dass andere Schüler/innen besser sind als sie selbst. Es gibt keinen signifikanten Unterschied zwischen den Gruppen. Item 23 bezieht sich dagegen auf die individuellen Empfindungen, dass andere Lernende besser sprechen können.

Item 23: Ich hatte immer das Gefühl, dass andere Schüler besser sprechen als ich.

\begin{tabular}{|c|c|c|c|c|c|c|}
\hline Gruppe & $\begin{array}{c}\text { starke Ab- } \\
\text { lehnung }\end{array}$ & Ablehnung & $\begin{array}{c}\text { Unent- } \\
\text { schieden }\end{array}$ & $\begin{array}{c}\text { Zustim- } \\
\text { mung }\end{array}$ & $\begin{array}{c}\text { starke Zu- } \\
\text { stimmung }\end{array}$ & $\begin{array}{c}\text { Mittelwert } \\
\text { (Standard- } \\
\text { abwei- } \\
\text { chung) }\end{array}$ \\
\hline $\begin{array}{c}\text { Magister- } \\
\text { Studium }\end{array}$ & 30 & 26 & 16 & 14 & 14 & $\begin{array}{c}2,56 \\
(1,41)\end{array}$ \\
\hline $\begin{array}{c}\text { Berufs- } \\
\text { Studium }\end{array}$ & 11 & 33 & 24 & 26 & 6 & 2,84 \\
\end{tabular}

Wenn man die Ergebnisse der Items 7 und 23 miteinander vergleicht, erkennt man fast identische Mittelwerte. Die Korrelationen betragen entsprechend $r=$ .68 in der Magistergruppe, $r=.65$ in der Berufsgruppe. In der Berufsgruppe überwiegen leicht diejenigen, die meinen, dass andere Lernende im Sprechen besser sind. Die Unterschiede zwischen den Gruppen sind aber statistisch nicht signifikant.

Item 31: Ich fürchtete, andere Schüler werden sich lustig über mich machen, wenn ich Deutsch spreche.

\begin{tabular}{|c|c|c|c|c|c|c|}
\hline Gruppe & $\begin{array}{c}\text { starke Ab- } \\
\text { lehnung }\end{array}$ & Ablehnung & $\begin{array}{c}\text { unent- } \\
\text { schieden }\end{array}$ & $\begin{array}{c}\text { Zustim- } \\
\text { mung }\end{array}$ & $\begin{array}{c}\text { starke Zu- } \\
\text { stimmung }\end{array}$ & $\begin{array}{c}\text { Mittelwert } \\
\text { (Standard- } \\
\text { abwei- } \\
\text { chung) }\end{array}$ \\
\hline $\begin{array}{c}\text { Magister- } \\
\text { Studium }\end{array}$ & 43 & 31 & 8 & 10 & 8 & $\begin{array}{c}2,07 \\
(1,27)\end{array}$ \\
\hline $\begin{array}{c}\text { Berufs- } \\
\text { Studium }\end{array}$ & 40 & 26 & 9 & 18 & 7 & 2,24 \\
& & $1,23)$ \\
\hline
\end{tabular}

Die Mittelwerte zeigen, dass die Gruppen sich in diesem Problem kaum unterscheiden. Die meisten Antworten widersprechen der angegebenen Meinung. Unter den Magisterstudierenden gibt es neun Personen, die dieser Meinung zustimmen. Die gemessenen Angstgefühle überschreiten bei allen Personen den Mittelwert der Gruppe mit 109,5 (79,9). Ähnlich verteilen sich die Werte 
bei der anderen Gruppe mit elf Personen ${ }^{15}$ bei $112,6(88,6)$. Es ist also offensichtlich, dass bei besonders ängstlichen Personen auch Befürchtungen vor eventuellen negativen Reaktionen der Mitlernenden auftauchen. Es konnte mit Hilfe dieses Untersuchungsinstruments nicht festgestellt werden, ob diese Befürchtungen der Lernenden nur auf ihren negativen Erwartungen oder aber auf wahren Vorerfahrungen basieren.

Item 13: Ich scheute mich davor, mich im Unterricht zu Wort zu melden.

\begin{tabular}{|c|c|c|c|c|c|c|}
\hline Gruppe & $\begin{array}{c}\text { starke Ab- } \\
\text { lehnung }\end{array}$ & Ablehnung & $\begin{array}{c}\text { Unent- } \\
\text { schieden }\end{array}$ & $\begin{array}{c}\text { Zustim- } \\
\text { mung }\end{array}$ & $\begin{array}{c}\text { starke Zu- } \\
\text { stimmung }\end{array}$ & $\begin{array}{c}\text { Mittelwert } \\
\text { (Standard- } \\
\text { abwei- } \\
\text { chung) }\end{array}$ \\
\hline $\begin{array}{c}\text { Magister- } \\
\text { Studium }\end{array}$ & 39 & 21 & 16 & 20 & 4 & $\begin{array}{c}2,27 \\
(1,28)\end{array}$ \\
\hline $\begin{array}{c}\text { Berufs- } \\
\text { Studium }\end{array}$ & 33 & 26 & 9 & 22 & 11 & 2,52 \\
& & $1,42)$ \\
\hline
\end{tabular}

Aus den Daten folgt, dass die Mehrheit der Studierenden in den beiden Gruppen keine Scheu spürt, sich im Unterricht zu Wort zu melden. Unter den Magisterstudierenden sind es 24\% (12 Personen), unter den Berufsstudierenden $33 \%$ (15 Personen), die solche Angstgefühle haben. Der Mittelwert auf der Gesamtskala der Angstgefühle beträgt bei diesen 27 Personen 108 Punkte (gleicher Wert in beiden Gruppen), liegt also viel höher als die gesamten Mittelwerte $(79,9 ; 88,6)$. In Item 14 werden die Probanden und Probandinnen gefragt, ob sie keine Angst spüren, wenn sie mit einem Muttersprachler oder einer Muttersprachlerin sprechen.

Item 14: Ich hätte keine Angst, wenn ich mit einem Muttersprachler sprechen würde.

\begin{tabular}{|c|c|c|c|c|c|c|}
\hline Gruppe & $\begin{array}{c}\text { starke Ab- } \\
\text { lehnung }\end{array}$ & Ablehnung & $\begin{array}{c}\text { unent- } \\
\text { schieden }\end{array}$ & $\begin{array}{c}\text { Zustim- } \\
\text { mung }\end{array}$ & $\begin{array}{c}\text { starke Zu- } \\
\text { stimmung }\end{array}$ & $\begin{array}{c}\text { Mittelwert } \\
\text { (Standard- } \\
\text { abwei- } \\
\text { chung) }\end{array}$ \\
\hline $\begin{array}{c}\text { Magister- } \\
\text { Studium }\end{array}$ & 12 & 23 & 20 & 25 & 20 & $2,82^{16}$ \\
\hline $\begin{array}{c}\text { Berufs- } \\
\text { Studium }\end{array}$ & 24 & 17 & 22 & 20 & 17 & 3,10 \\
& & & $1,43)$ \\
\hline
\end{tabular}

15 In dieser Gruppe gibt es nur eine Person, deren Gesamtergebnis auf der Angstskala unter dem Mittelwert liegt.

16 Umgekehrt gerechnet (starke Ablehnung 5 - starke Zustimmung 1). 
Die Tabelle zeigt, dass in beiden Gruppen keine entschiedenen Meinungsmehrheiten feststellbar sind. In der Gruppe der Berufsstudierenden überwiegen leicht die ablehnenden Antworten (41\%). Sehr groß ist auch die Anzahl der Unentschiedenen in beiden Gruppen. Diese Verteilung der Werte ist darauf zurückzuführen, dass die Proband(inn)en möglicherweise selten die Gelegenheit hatten, mit einem/einer Deutschen zu sprechen, so dass sie keine eindeutige Antwort geben konnten. Es ist auch eine Tatsache, dass viele Studienanfänger Angstgefühle oder zumindest Hemmungen zeigen, wenn sie im Unterricht mit einem Muttersprachler/einer Muttersprachlerin sprechen sollen. Item 32 bezieht sich auf das Problem des Lernortes.

Item 32: Es scheint mir, dass ich mich lockerer fühlen würde, wenn ich in Deutschland lernen würde.

\begin{tabular}{|c|c|c|c|c|c|c|}
\hline Gruppe & $\begin{array}{c}\text { starke Ab- } \\
\text { lehnung }\end{array}$ & Ablehnung & $\begin{array}{c}\text { unent- } \\
\text { schieden }\end{array}$ & $\begin{array}{c}\text { Zustim- } \\
\text { mung }\end{array}$ & $\begin{array}{c}\text { starke Zu- } \\
\text { stimmung }\end{array}$ & $\begin{array}{c}\text { Mittelwert } \\
\text { (Standard- } \\
\text { abwei- } \\
\text { chung) }\end{array}$ \\
\hline $\begin{array}{c}\text { Magister- } \\
\text { Studium }\end{array}$ & 6 & 14 & 29 & 27 & 24 & $\begin{array}{c}2,500^{17} \\
(1,17)\end{array}$ \\
\hline $\begin{array}{c}\text { Berufs- } \\
\text { Studium }\end{array}$ & 6 & 2 & 35 & 35 & 22 & $\begin{array}{c}2,36 \\
(1,06)\end{array}$ \\
\hline
\end{tabular}

Interessanterweise stehen sogar 20\% der Magisterstudierenden dieser Aussage skeptisch gegenüber (in der Berufsgruppe nur $8 \%$ ). Viele der Befragten sind unentschieden. Wenn man die Korrelation zwischen den Items 14 und 32 gemessen hat, kann man erkennen, dass sie in der Berufsgruppe sehr schwach ist $(r=.19)$. In der Magistergruppe ist sie sogar negativ $(r=-.21)$. Daraus resultiert, dass diese zwei Punkte keine Abhängigkeitsbeziehungen aufweisen.

\section{Verstehensangst}

Bei diesem Faktor ging es darum, ob die Verstehensprobleme im Unterricht Angstgefühle auslösen konnten. Die Ursachen verschiedener fremdsprachlicher Ängste liegen nämlich oft darin, dass die Lernenden nicht alles verstehen oder aber sich keine Mühe geben, die Verstehensprozesse auf der Ebene des Kontextes und nicht der einzelnen Wörter herzustellen. Die Verstehensangst wird mit den Items 4, 15 und 29 gemessen.

17 Umgekehrt gerechnet (starke Ablehnung 5 - starke Zustimmung 1). 
Angstgefühle und deren mögliche Auswirkungen

Item 4: Ich war aufgeregt, wenn ich nicht verstehen konnte, was der Lehrer sagte.

\begin{tabular}{|c|c|c|c|c|c|c|}
\hline Gruppe & $\begin{array}{c}\text { starke } \\
\text { Ableh- } \\
\text { nung }\end{array}$ & $\begin{array}{c}\text { Ableh- } \\
\text { nung }\end{array}$ & $\begin{array}{c}\text { unent- } \\
\text { schieden }\end{array}$ & $\begin{array}{c}\text { Zustim- } \\
\text { mung }\end{array}$ & $\begin{array}{c}\text { starke Zu- } \\
\text { stimmung }\end{array}$ & $\begin{array}{c}\text { Mittelwert } \\
\text { (Standard- } \\
\text { abwei- } \\
\text { chung) }\end{array}$ \\
\hline $\begin{array}{c}\text { Magister- } \\
\text { Studium }\end{array}$ & 16 & 41 & 8 & 21 & 14 & $\begin{array}{c}2,76 \\
(1,33)\end{array}$ \\
\hline $\begin{array}{c}\text { Berufs- } \\
\text { Studium }\end{array}$ & 13 & 18 & 11 & 38 & 20 & $\begin{array}{c}3,33 \\
(1,34)\end{array}$ \\
\hline
\end{tabular}

Aus der Tabelle ist ersichtlich, dass die Berufsstudierenden häufiger aufgeregt sind, wenn sie etwas nicht verstehen können, was der Lehrer sagt. Über die Mehrheit (58\%) stimmt dieser Meinung zu. Die statistische Analyse ergibt aber keine signifikanten Unterschiede zwischen beiden Gruppen.

Item 15: Ich ärgerte mich, wenn ich nicht verstehen konnte, welche Fehler der Lehrer bei mir korrigierte.

\begin{tabular}{|c|c|c|c|c|c|c|}
\hline Gruppe & $\begin{array}{c}\text { starke Ab- } \\
\text { lehnung }\end{array}$ & Ablehnung & $\begin{array}{c}\text { unent- } \\
\text { schieden }\end{array}$ & $\begin{array}{c}\text { Zustim- } \\
\text { mung }\end{array}$ & $\begin{array}{c}\text { starke Zu- } \\
\text { stimmung }\end{array}$ & $\begin{array}{c}\text { Mittelwert } \\
\text { (Standard- } \\
\text { abwei- } \\
\text { chung) }\end{array}$ \\
\hline $\begin{array}{c}\text { Magister- } \\
\text { Studium }\end{array}$ & 16 & 28 & 18 & 32 & 6 & $\begin{array}{c}2,84 \\
(1,21)\end{array}$ \\
\hline $\begin{array}{c}\text { Berufs- } \\
\text { Studium }\end{array}$ & 13 & 15 & 28 & 35 & 4 & 3,10 \\
& & $1,17)$ \\
\hline
\end{tabular}

Auch bei diesem Item erreichen die Ja-Antworten fast den gleichen Wert (38\%, 39\%). In der Gruppe der Berufsstudierenden ist die Prozentzahl der Unentschiedenen um 10\% größer als bei den Magisterstudierenden. In der Gruppe der Magisterstudierenden korrelieren diese Werte mit den Werten von Item 4 auf einem mittleren Niveau $(r=.50)$. In der Berufsgruppe ist diese Korrelation niedrig $(\mathrm{r}=.29)$. 
Krzysztof Nerlicki

Item 29: Ich ärgerte mich, wenn ich nicht alle Wörter verstehen konnte.

\begin{tabular}{|c|c|c|c|c|c|c|}
\hline Gruppe & $\begin{array}{c}\text { starke Ab- } \\
\text { lehnung }\end{array}$ & Ablehnung & $\begin{array}{c}\text { unent- } \\
\text { schieden }\end{array}$ & $\begin{array}{c}\text { Zustim- } \\
\text { mung }\end{array}$ & $\begin{array}{c}\text { starke Zu- } \\
\text { stimmung }\end{array}$ & $\begin{array}{c}\text { Mittelwert } \\
\text { (Standard- } \\
\text { abwei- } \\
\text { chung) }\end{array}$ \\
\hline $\begin{array}{c}\text { Magister- } \\
\text { Studium }\end{array}$ & 22 & 36 & 14 & 24 & 4 & $\begin{array}{c}2,52 \\
(1,19)\end{array}$ \\
\hline $\begin{array}{c}\text { Berufs- } \\
\text { Studium }\end{array}$ & 19 & 30 & 19 & 19 & 11 & $\begin{array}{c}2,71 \\
(1,29)\end{array}$ \\
\hline
\end{tabular}

In dieser Frage vertreten die Proband(inn)en fast die gleiche Meinung. Die Korrelationen zwischen den Items 4 und 29 sind auch nicht hoch. Sie betragen jeweils $r=.44$ und $r=.41$. Aus der Analyse der obigen drei Faktoren kann man schließen, dass die Germanistikstudierenden das Verstehen nicht stark als angstauslösende Quelle betrachten. Dies kann darauf zurückgeführt werden, dass die rezeptive Kompetenz der Lernenden verhältnismäßig hoch ist. Andererseits muss eingeräumt werden, dass die Lehrersprache im Unterricht sicherlich auch den Umständen angepasst war, z. B. bezüglich des Tempos und der Artikulation, so dass möglichst ungestörte Wahrnehmungsprozesse der lautlichen Substanz gewährleistet waren.

\section{Prüfungsangst}

Dieser Faktor bezieht sich auf Angstgefühle, die im Zusammenhang mit Fremdbeurteilung entstehen können, insbesondere bei Klausurarbeiten und Prüfungen, aber auch bei eventuellen Misserfolgen. Für die Untersuchung ist interessant, welche Meinung die Probanden/innen über schulische Klassenarbeiten (z.B. Tests) haben. Die Items 8, 10, und 21 versuchen unterschiedliche Aspekte der Prüfungsangst zu messen. Zum einen geht es um das allgemeine Befinden der Befragten während der Tests (Item 8), zum anderen handelt es sich auch um das Verhältnis zwischen dem Arbeitsaufwand während der Vorbereitungen auf einen Test und der inneren Sicherheit, dass man genug gelernt hat (Item 21). Schließlich erscheint auch die Frage nach der Einstellung der Probanden/innen zu möglichen negativen Konsequenzen der Misserfolge im Deutschunterricht (Item 10) legitim. 
Item 8: Ich fühlte mich gewöhnlich unter Druck während eines Tests oder einer Klausur in Deutsch.

\begin{tabular}{|c|c|c|c|c|c|c|}
\hline Gruppe & $\begin{array}{c}\text { starke Ab- } \\
\text { lehnung }\end{array}$ & Ablehnung & $\begin{array}{c}\text { unent- } \\
\text { schieden }\end{array}$ & $\begin{array}{c}\text { Zustim- } \\
\text { mung }\end{array}$ & $\begin{array}{c}\text { Starke Zu- } \\
\text { stimmung }\end{array}$ & $\begin{array}{c}\text { Mittelwert } \\
\text { (Standard- } \\
\text { abwei- } \\
\text { chung) }\end{array}$ \\
\hline $\begin{array}{c}\text { Magister- } \\
\text { Studium }\end{array}$ & 6 & 14 & 18 & 39 & 23 & $\begin{array}{c}2,39^{18} \\
(1,16)\end{array}$ \\
\hline $\begin{array}{c}\text { Berufs- } \\
\text { Studium }\end{array}$ & 22 & 13 & 17 & 35 & 12 & $\begin{array}{c}2,95 \\
(1,38)\end{array}$ \\
\hline
\end{tabular}

Vergleicht man die prozentualen Werte, so scheinen die Berufsstudierenden $(47 \%)$ in einer Klausursituation weniger unter Druck zu sein als ihre Kommiliton(inn)en im Magisterstudium (62\%). Die Unterschiede zwischen den Gruppen sind aber statistisch nicht signifikant.

Item 21: Je mehr ich für eine Klausur lernte, desto weniger wusste ich.

\begin{tabular}{|c|c|c|c|c|c|c|}
\hline Gruppe & $\begin{array}{c}\text { starke Ab- } \\
\text { lehnung }\end{array}$ & Ablehnung & $\begin{array}{c}\text { unent- } \\
\text { schieden }\end{array}$ & $\begin{array}{c}\text { Zustim- } \\
\text { mung }\end{array}$ & $\begin{array}{c}\text { Starke Zu- } \\
\text { stimmung }\end{array}$ & $\begin{array}{c}\text { Mittelwert } \\
\text { (Standard- } \\
\text { abwei- } \\
\text { chung) }\end{array}$ \\
\hline $\begin{array}{c}\text { Magister- } \\
\text { Studium }\end{array}$ & 47 & 31 & 10 & 8 & 4 & $\begin{array}{c}1,90 \\
(1,11)\end{array}$ \\
\hline $\begin{array}{c}\text { Berufs- } \\
\text { Studium }\end{array}$ & 39 & 28 & 6 & 22 & 4 & $\begin{array}{c}2,23 \\
(1,30)\end{array}$ \\
\hline
\end{tabular}

Wie bei Item 8 unterscheiden sich die prozentualen Werte zwischen den verglichenen Gruppen, sie sind aber statistisch nicht signifikant. Die Mehrheit in beiden Gruppen stimmt dieser Meinung nicht zu (78\%, 67\%), aber unter den Berufsstudierenden sehen sich $26 \%$ (12 Personen) der Befragten mit solchen Gefühlen konfrontiert. Der Mittelwert des Angstgrades beträgt bei diesen 12 Personen 111 Punkte, ist also verhältnismäßig hoch. In der Gruppe der Magisterstudierenden gibt es sechs Personen, deren Mittelwert nicht so hoch ist (95 Punkte). Die Ergebnisse zeugen davon, dass dieser Faktor in der Berufsgruppe eng mit anderen Angstgefühlen verbunden ist.

18 Umgekehrt gerechnet (starke Ablehnung 5 - starke Zustimmung 1). 
Krzysztof Nerlicki

Item 10: Ich machte mir Sorgen um die Konsequenzen meiner Misserfolge im Deutschunterricht.

\begin{tabular}{|c|c|c|c|c|c|c|}
\hline Gruppe & $\begin{array}{c}\text { starke Ab- } \\
\text { lehnung }\end{array}$ & Ablehnung & $\begin{array}{c}\text { unent- } \\
\text { schieden }\end{array}$ & $\begin{array}{c}\text { Zustim- } \\
\text { mung }\end{array}$ & $\begin{array}{c}\text { starke Zu- } \\
\text { stimmung }\end{array}$ & $\begin{array}{c}\text { Mittelwert } \\
\text { (Standard- } \\
\text { abwei- } \\
\text { chung) }\end{array}$ \\
\hline $\begin{array}{c}\text { Magister- } \\
\text { Studium }\end{array}$ & 23 & 21 & 16 & 23 & 16 & $\begin{array}{c}2,86 \\
(1,42)\end{array}$ \\
\hline $\begin{array}{c}\text { Berufs- } \\
\text { Studium }\end{array}$ & 15 & 22 & 22 & 25 & 15 & $\begin{array}{c}3,02 \\
(1,32)\end{array}$ \\
\hline
\end{tabular}

Die Studierenden haben fast gleiche Meinungen zu diesem Item. Die Antworten (Zustimmung, Ablehnung) verteilen sich etwa um $40 \%$. Man kann deutlich bemerken, dass diejenigen, die dieser Meinung zustimmen, auch viel ängstlicher sind (Mittelwerte: Magisterstudenten - 96 Punkte; Berufsstudenten - 107 Punkte) als diejenigen, die anderer Ansicht sind (Mittelwerte: Magisterstudenten - 64 Punkte; Berufsstudenten - 71 Punkte).

\section{Angstfaktoren, die innerhalb oder außerhalb des Klassenraumes entstehen}

Weiterhin ist überprüft worden, wie die Befragten andere Faktoren, die mit den unterrichtlichen Tätigkeiten nicht unmittelbar zusammenhängen, im Kontext möglicher Angstgefühle einschätzen. Diese Faktoren haben einen allgemeinen Charakter und sind durch die Spezifik des schulischen Lernens geprägt. Die innerunterrichtlichen Faktoren werden mit den Items 6, 12, 25, 26, die äußeren Faktoren mit den Items 5, 11, 16, 17, 22, 28 gemessen.

Item 6: Im Deutschunterricht dachte ich oft über Dinge nach, die mit der Stunde nichts zu tun hatten.

\begin{tabular}{|c|c|c|c|c|c|c|}
\hline Gruppe & $\begin{array}{c}\text { starke Ab- } \\
\text { lehnung }\end{array}$ & Ablehnung & $\begin{array}{c}\text { unent- } \\
\text { schieden }\end{array}$ & $\begin{array}{c}\text { Zustim- } \\
\text { mung }\end{array}$ & $\begin{array}{c}\text { starke Zu- } \\
\text { stimmung }\end{array}$ & $\begin{array}{c}\text { Mittelwert } \\
\text { (Standard- } \\
\text { abwei- } \\
\text { chung })\end{array}$ \\
\hline $\begin{array}{c}\text { Magister- } \\
\text { Studium }\end{array}$ & 22 & 31 & 23 & 16 & 8 & $\begin{array}{c}2,56 \\
(1,22)\end{array}$ \\
\hline $\begin{array}{c}\text { Berufs- } \\
\text { Studium }\end{array}$ & 24 & 37 & 19 & 11 & 9 & 2,43 \\
$(1,22)$
\end{tabular}


Für die Mehrheit der Befragten trifft diese Aussage nicht zu. Es ist aber zu verzeichnen, dass viele der Befragten (um 20\%) keine Meinung haben. Man kann nicht sagen, ob dieser Faktor mit dem Gesamtwert der Angstgefühle zusammenhängt. Unter den Magisterstudierenden, die diese Ansicht teilen (12 Personen), beträgt der Durchschnittswert 79 Punkte. Bei den Berufsstudierenden sind es neun Personen - ihr Durchschnittswert liegt bei 94 Punkten. Man kann also nicht den Schluss ziehen, dass besonders ängstliche Lernende über andere Dinge im Deutschunterricht nachdenken.

Item 12: Im Deutschunterricht war ich so gestresst, dass ich alles vergessen habe, was ich schon wusste.

\begin{tabular}{|c|c|c|c|c|c|c|}
\hline Gruppe & $\begin{array}{c}\text { starke Ab- } \\
\text { lehnung }\end{array}$ & Ablehnung & $\begin{array}{c}\text { unent- } \\
\text { schieden }\end{array}$ & $\begin{array}{c}\text { Zustim- } \\
\text { mung }\end{array}$ & $\begin{array}{c}\text { starke Zu- } \\
\text { stimmung }\end{array}$ & $\begin{array}{c}\text { Mittelwert } \\
\text { (Standard- } \\
\text { abwei- } \\
\text { chung) }\end{array}$ \\
\hline $\begin{array}{c}\text { Magister- } \\
\text { Studium }\end{array}$ & 63 & 23 & 6 & 8 & 0 & $\begin{array}{c}1,58 \\
(0,92)\end{array}$ \\
\hline $\begin{array}{c}\text { Berufs- } \\
\text { Studium }\end{array}$ & 37 & 35 & 6 & 13 & 9 & $\begin{array}{c}2,21 \\
(1,31)\end{array}$ \\
\hline
\end{tabular}

Betrachtet man die Mittelwerte, so ist festzustellen, dass die Gruppe im Magisterstudium eine überwiegend ablehnende Meinung vertritt $(86 \%)$ und die Antworten nicht so stark gestreut sind. Sichtbar ist hingegen, dass in der Gruppe der Berufsstudierenden 22\% (10 Personen) der Befragten Angstgefühle eingeräumt haben (in der ersten Gruppe vier Personen). Festzustellen ist auch, dass diese 14 Personen recht hohe Gesamtergebnisse auf der Angstskala erreicht haben. Die Mittelwerte innerhalb dieser Gruppen betragen 110 bzw. 117 Punkte. Der Unterschied zwischen den Gruppen ist statistisch signifikant $\left(\chi^{2}=10,1, \mathrm{df}=4, \mathrm{p}<0,01\right)$.

Item 25: Der Deutschunterricht verlief so schnell, dass ich fürchtete, ich könnte nicht mithalten.

\begin{tabular}{|c|c|c|c|c|c|c|}
\hline Gruppe & $\begin{array}{c}\text { starke Ab- } \\
\text { lehnung }\end{array}$ & Ablehnung & $\begin{array}{c}\text { unent- } \\
\text { schieden }\end{array}$ & $\begin{array}{c}\text { Zustim- } \\
\text { mung }\end{array}$ & $\begin{array}{c}\text { starke Zu- } \\
\text { stimmung }\end{array}$ & $\begin{array}{c}\text { Mittelwert } \\
\text { (Standard- } \\
\text { abwei- } \\
\text { chung) }\end{array}$ \\
\hline $\begin{array}{c}\text { Magister- } \\
\text { Studium }\end{array}$ & 45 & 29 & 20 & 4 & 2 & $\begin{array}{c}1,88 \\
(0,99)\end{array}$ \\
\hline $\begin{array}{c}\text { Berufs- } \\
\text { Studium }\end{array}$ & 26 & 43 & 15 & 11 & 4 & 2,23 \\
$(1,09)$
\end{tabular}


In der Gruppe der Berufsstudierenden ist die Befürchtung, im Unterricht nicht mithalten zu können, um einige Prozentpunkte höher (6\%-15\%), aber wegen der relativ geringen Befragtenzahl ist dieser Unterschied nicht bedeutsam. Tatsache ist, dass die meisten Personen, die dieser Aussage zustimmen, auch Angstgefühle im Gesamtwert aufweisen (Magisterstudierende - 109 Punkte; Berufsstudierende - 121 Punkte).

Item 26: Im Deutschunterricht fühlte ich mich gehemmter und aufgeregter als in anderen Stunden.

\begin{tabular}{|c|c|c|c|c|c|c|}
\hline Gruppe & $\begin{array}{c}\text { starke Ab- } \\
\text { lehnung }\end{array}$ & Ablehnung & $\begin{array}{c}\text { unent- } \\
\text { schieden }\end{array}$ & $\begin{array}{c}\text { Zustim- } \\
\text { mung }\end{array}$ & $\begin{array}{c}\text { starke Zu- } \\
\text { stimmung }\end{array}$ & $\begin{array}{c}\text { Mittelwert } \\
\text { (Standard- } \\
\text { abwei- } \\
\text { chung) }\end{array}$ \\
\hline $\begin{array}{c}\text { Magister- } \\
\text { Studium }\end{array}$ & 57 & 23 & 10 & 8 & 2 & $\begin{array}{c}1,74 \\
(1,05)\end{array}$ \\
\hline $\begin{array}{c}\text { Berufs- } \\
\text { Studium }\end{array}$ & 41 & 37 & 9 & 9 & 4 & $\begin{array}{c}1,97 \\
(1,12)\end{array}$ \\
\hline
\end{tabular}

Auch in diesem Punkt ist die entschiedene Mehrheit mit dieser Meinung nicht einverstanden. Diejenigen, die sich im Deutschunterricht gehemmter und aufgeregter als in anderen Fächern fühlen, zeichnen sich insgesamt durch verhältnismäßig große Angstgefühle aus (Magisterstudierende: 5 Personen, durchschnittlich 111 Punkte; Berufsstudierende: 6 Personen, durchschnittlich 123 Punkte). In Item 5 werden die Probanden/innen nach der Häufigkeit des Deutschunterrichts gefragt.

Item 5: Ich hätte nichts dagegen, häufiger am Deutschunterricht teilnehmen zu müssen.

\begin{tabular}{|c|c|c|c|c|c|c|}
\hline Gruppe & $\begin{array}{c}\text { starke Ab- } \\
\text { lehnung }\end{array}$ & Ablehnung & $\begin{array}{c}\text { unent- } \\
\text { schieden }\end{array}$ & $\begin{array}{c}\text { Zustim- } \\
\text { mung }\end{array}$ & $\begin{array}{c}\text { starke Zu- } \\
\text { stimmung }\end{array}$ & $\begin{array}{c}\text { Mittelwert } \\
\text { (Standard- } \\
\text { abwei- } \\
\text { chung) }\end{array}$ \\
\hline $\begin{array}{c}\text { Magister- } \\
\text { Studium }\end{array}$ & 2 & 8 & 8 & 31 & 51 & $\begin{array}{c}1,788^{19} \\
(1,02)\end{array}$ \\
\hline $\begin{array}{c}\text { Berufs- } \\
\text { Studium }\end{array}$ & 13 & 9 & 9 & 19 & 50 & 2,15 \\
& & $1,46)$ \\
\hline
\end{tabular}

Bei den Magisterstudierenden hätte die Mehrheit nichts dagegen, mehr Deutschunterricht zu haben. Bei den Berufsstudierenden ist diese Mehrheit

19 Umgekehrt gerechnet (starke Ablehnung 5 - starke Zustimmung 1). 
auch deutlich, aber es gibt auch $22 \%$ der Befragten, die dieser Meinung skeptisch gegenüberstehen.

Item 11: Ich konnte nicht verstehen, warum andere Lerner sich so viele Sorgen um Deutsch machten.

\begin{tabular}{|c|c|c|c|c|c|c|}
\hline Gruppe & $\begin{array}{c}\text { starke Ab- } \\
\text { lehnung }\end{array}$ & Ablehnung & $\begin{array}{c}\text { unent- } \\
\text { schieden }\end{array}$ & $\begin{array}{c}\text { Zustim- } \\
\text { mung }\end{array}$ & $\begin{array}{c}\text { starke Zu- } \\
\text { stimmung }\end{array}$ & $\begin{array}{c}\text { Mittelwert } \\
\text { (Standard- } \\
\text { abwei- } \\
\text { chung) }\end{array}$ \\
\hline $\begin{array}{c}\text { Magister- } \\
\text { Studium }\end{array}$ & 20 & 26 & 20 & 20 & 14 & $3,18^{20}$ \\
& 17 & 26 & 33 & 15 & 9 & 3,28 \\
\hline $\begin{array}{c}\text { Berufs- } \\
\text { Studium }\end{array}$ & 17 & & $1,35)$ \\
\hline
\end{tabular}

Diese Frage berührt das Problem der Empathie. Es ist davon auszugehen, dass ängstlichere Personen sich auch leichter in die Situation anderer Personen versetzen können, die mit Deutsch Probleme haben. Überprüft worden ist, ob diejenigen Befragten, die dieser Aussage nicht zustimmen, deutlich weniger ängstlich sind als diejenigen, die diese Meinung bejahen. In der Magistergruppe beträgt das Verhältnis 64 (Ablehnung) zu 87 (Zustimmung) Punkte, in der Berufsgruppe 72 zu 96 Punkte. Man kann also annehmen, dass ängstlichere Personen auch das Empathiegefühl, andere mit solchen vergleichbaren Problemen verstehen zu können, stärker entwickelt haben.

Item 16: Ich war sogar dann beunruhigt, wenn ich für den Unterricht gut vorbereitet war.

\begin{tabular}{|c|c|c|c|c|c|c|}
\hline Gruppe & $\begin{array}{c}\text { starke Ab- } \\
\text { lehnung }\end{array}$ & Ablehnung & $\begin{array}{c}\text { unent- } \\
\text { schieden }\end{array}$ & $\begin{array}{c}\text { Zustim- } \\
\text { mung }\end{array}$ & $\begin{array}{c}\text { starke Zu- } \\
\text { stimmung }\end{array}$ & $\begin{array}{c}\text { Mittelwert } \\
\text { (Standard- } \\
\text { abwei- } \\
\text { chung) }\end{array}$ \\
\hline $\begin{array}{c}\text { Magister- } \\
\text { Studium }\end{array}$ & 37 & 27 & 8 & 25 & 2 & $\begin{array}{c}2,27 \\
(1,26)\end{array}$ \\
\hline $\begin{array}{c}\text { Berufs- } \\
\text { Studium }\end{array}$ & 35 & 26 & 6 & 22 & 11 & 2,47 \\
\end{tabular}

Aus der Tabelle geht hervor, dass die Prozentwerte in beiden Gruppen fast gleich sind. Die Mehrheit der Befragten stimmt mit dieser Meinung nicht überein. Diejenigen, die diese Meinung teilen, gehören wieder zu den ängstli-

20 Umgekehrt gerechnet (starke Ablehnung 5 - starke Zustimmung 1). 
cheren Personen (Magisterstudierende: 14 Personen, durchschnittlich 101 Punkte; Berufsstudierende: 15 Personen, durchschnittlich 113 Punkte).

Item 17: Oft würde ich gerne den Deutschunterricht schwänzen.

\begin{tabular}{|c|c|c|c|c|c|c|}
\hline Gruppe & $\begin{array}{c}\text { starke Ab- } \\
\text { lehnung }\end{array}$ & Ablehnung & $\begin{array}{c}\text { unent- } \\
\text { schieden }\end{array}$ & $\begin{array}{c}\text { Zustim- } \\
\text { mung }\end{array}$ & $\begin{array}{c}\text { starke Zu- } \\
\text { stimmung }\end{array}$ & $\begin{array}{c}\text { Mittelwert } \\
\text { (Standard- } \\
\text { abwei- } \\
\text { chung) }\end{array}$ \\
\hline $\begin{array}{c}\text { Magister- } \\
\text { Studium }\end{array}$ & 35 & 33 & 12 & 16 & 4 & 2,19 \\
\hline $\begin{array}{c}\text { Berufs- } \\
\text { Studium }\end{array}$ & 37 & 26 & 15 & 15 & 6 & 2,28 \\
\end{tabular}

Die Werte verteilen sich fast gleich. In jeder Gruppe gibt es je 10 Personen, die dieser Meinung sind. Ihre Gesamtwerte auf der Angstskala liegen auch über 100 Punkten und betragen entsprechend 102:73 (ablehnende Antwort) und 111:68 (ablehnende Antwort) Punkte.

Item 22: Ich musste nicht dazu gezwungen werden, Deutsch zu lernen.

\begin{tabular}{|c|c|c|c|c|c|c|}
\hline Gruppe & $\begin{array}{c}\text { starke Ab- } \\
\text { lehnung }\end{array}$ & Ablehnung & $\begin{array}{c}\text { unent- } \\
\text { schieden }\end{array}$ & $\begin{array}{c}\text { Zustim- } \\
\text { mung }\end{array}$ & $\begin{array}{c}\text { starke Zu- } \\
\text { stimmung }\end{array}$ & $\begin{array}{c}\text { Mittelwert } \\
\text { (Standard- } \\
\text { abwei- } \\
\text { chung) }\end{array}$ \\
\hline $\begin{array}{c}\text { Magister- } \\
\text { Studium }\end{array}$ & 2 & 2 & 2 & 28 & 66 & $\begin{array}{c}1,46 \\
(, 81)\end{array}$ \\
\hline $\begin{array}{c}\text { Berufs- } \\
\text { Studium }\end{array}$ & 4 & 9 & 11 & 26 & 50 & $\begin{array}{c}1,91 \\
(1,17)\end{array}$ \\
\hline
\end{tabular}

Aus der Tabelle ist zu schließen, dass die Berufsstudierenden nur zu $76 \%$ dieser Aussage zustimmen (die Magisterstudierenden zu 94\%). Die Unterschiede zwischen den Gruppen sind statistisch nicht signifikant. 
Angstgefühle und deren mögliche Auswirkungen

Item 28: Wenn ich zum Deutschunterricht ging, fühlte ich mich sehr sicher und entspannt.

\begin{tabular}{|c|c|c|c|c|c|c|}
\hline Gruppe & $\begin{array}{c}\text { starke Ab- } \\
\text { lehnung }\end{array}$ & Ablehnung & $\begin{array}{c}\text { unent- } \\
\text { schieden }\end{array}$ & $\begin{array}{c}\text { Zustim- } \\
\text { mung }\end{array}$ & $\begin{array}{c}\text { starke Zu- } \\
\text { stimmung }\end{array}$ & $\begin{array}{c}\text { Mittelwert } \\
\text { (Standard- } \\
\text { abwei- } \\
\text { chung) }\end{array}$ \\
\hline $\begin{array}{c}\text { Magister- } \\
\text { Studium }\end{array}$ & 18 & 14 & 21 & 29 & 18 & $\begin{array}{c}2,84^{21} \\
(1,36)\end{array}$ \\
\hline $\begin{array}{c}\text { Berufs- } \\
\text { Studium }\end{array}$ & 13 & 27 & 13 & 31 & 16 & $\begin{array}{c}2,91 \\
(1,32)\end{array}$ \\
\hline
\end{tabular}

Zwischen den beiden Gruppen sind kaum Unterschiede festsellbar, und die Stimmen für und gegen diese Meinung verteilen sich fast je zur Hälfte. Auch in diesem Fall zeichnet sich die Gruppe derjenigen Studierenden, die eine ablehnende Antwort gibt, durch höhere Angstwerte auf der Gesamtskala aus (Magisterstudierende 94:67; Berufsstudierende 112:68).

\section{Diskussion}

Das Ziel der dargestellten empirischen Untersuchung ist es festzustellen, ob Studienanfänger der Germanistik auf Grund ihrer bisherigen Lernerfahrungen Angstgefühle haben, und wenn ja, welcher Art diese Angstgefühle sind. Die Analyse umfasst zwei Gruppen von Studierenden - im fünfjährigen Magisterstudium und im dreijährigen Berufsstudium. Da die Probandengruppen verhältnismäßig klein sind, ist die Untersuchung als Fallanalyse zu betrachten. Die Mittelwerte auf der Gesamtskala des Angstgrades betragen 79,9 (Magisterstudierende) und 88,6 (Berufsstudierende) von 165 Gesamtpunkten. Statistisch gesehen sind die Unterschiede nicht signifikant, wobei jedoch die Tendenz erkennbar ist, dass die Studierenden im Berufsstudium immer um einige Prozentpunkte höhere Werte auf der Gesamtskala aufweisen. Es wäre natürlich interessant herauszufinden, ob diese höheren Werte z.B. mit dem Kompetenzniveau der Studierenden korrelieren. Man kann die Behauptung wagen, dass Studierende im Magisterstudium sprachlich kompetenter sind als ihre Kommilitonen/innen, die das dreijährige Studium an einer anderen Hochschule als an der Universität anfangen, was häufiger zu Ängsten führen könnte. Wenn man alle Antworten in den beiden Gruppen zusammenrechnet, dann erweisen sich die Unterschiede als statistisch signifikant $\left(\chi^{2}=14,66\right.$, df $\left.=4, p<0,01\right)$.

21 Umgekehrt gerechnet (starke Ablehnung 5 - starke Zustimmung 1). 
In den untersuchten Gruppen lassen sich einige interessante Tendenzen, insbesondere hinsichtlich der Sprechangst, festhalten. So spielen bei den Studierenden die Umstände der Sprechsituationen eine Rolle. Da diese Situationen für die meisten Lernenden vor allem vor dem Hintergrund des schulischen Kontextes eingeschätzt werden, sind bei dieser Beurteilung die bisherigen unterrichtlichen Erfahrungen von Bedeutung. Erwähnt werden Faktoren wie Sprechen in und vor der Gruppe, Interaktionen (auch Abfragen) mit den Lehrenden und Einstellungen zu Fehlern (vgl. FISCHER 2005). Erkennbar ist, dass die Lernenden es im Allgemeinen nicht besonders mögen, vor die Klasse zu treten und zu sprechen. Viele ängstliche Lernende sind sehr stark vom Lernumfeld abhängig, so dass ihre Angstgefühle daher rühren, wie sie von anderen beurteilt werden (z.B. besteht die Gefahr, ausgelacht zu werden). So sind auch Vergleiche der eigenen Person mit anderen für manche eher angstauslösend. Einen signifikanten Unterschied lässt auch das Problem des Abfragens erkennen. Studierende im Berufsstudium stimmen häufiger zu, Angstgefühle zu haben, wenn sie wussten, dass sie abgefragt werden. Es kann außerdem festgestellt werden, dass z.B. Fehlerkorrekturen seitens der Lehrenden weniger zur Angst führen als die Tatsache, dass man persönlich immer wieder Fehler begeht. In der Gruppe der Berufsstudierenden ist die Überzeugung verbreitet, dass die Menge von nötigen Regelkenntnissen, um sprechen zu können, eher lernhemmend wirken kann. Es ist nicht festzustellen, dass unerwartete Fragen besonders stark auf eventuelle Ängste Einfluss haben. Im Bereich der Verstehensangst haben vor allem Berufsstudierende Angstgefühle, wenn sie etwas nicht verstanden haben. Sie fühlen sich während einer Klausur (eines Tests) weniger entspannt als ihre Kommilitonen/innen im Magisterstudium. Unter den innerunterrichtlichen Faktoren hat das Item 12 signifikante Unterschiede ergeben. So geben $22 \%$ der Berufsstudierenden an, auf Grund von Stress alles im Unterricht vergessen zu haben. In der Gruppe der äußeren Faktoren, die Angstgefühle auslösen können, ist als Ergebnis festzuhalten, dass alle überdurchschnittlich Ängstlichen auch keine Lust auf mehr Deutschunterricht haben; sie haben trotz guter Vorbereitung Angst und erwägen, den Unterricht zu schwänzen. Unter den Magisterstudierenden gibt es 12 Personen (11 Frauen, 1 Mann), die 100 und mehr Punkte (von 165) auf der Gesamtskala der Ängstlichkeit erreicht haben. In der Gruppe der Berufsstudierenden sind es 16 Personen (14 Frauen, 2 Männer). Feststellbar ist, dass unter diesen 28 Personen das Item 22 (,Ich musste nicht dazu gezwungen werden, Deutsch zu lernen.“) am seltensten Angstgefühle auslöst. Es folgen die Items 5 („,Ich hätte nichts dagegen, häufiger am Deutschunterricht teilnehmen zu müssen.“) und 32 („Es scheint mir, dass ich mich lockerer fühlen würde, wenn ich in Deutschland 
lernen würde."). In der Gruppe der ängstlichen Magisterstudierenden hat das Item 1 (,Beim Deutschsprechen in der Klasse habe ich mich nie ganz sicher gefühlt.") den höchsten Mittelwert (4,33). In der Gruppe der Berufsstudierenden sind dies die Items 4 (,Ich war aufgeregt, wenn ich nicht verstehen konnte, was der Lehrer sagte.“) und 24 (,Ich fühlte mich unsicher, wenn ich vor die Klasse treten und sprechen sollte."; beide 4,37).

Die Probanden bilden eine besondere Gruppe von Deutschlernenden. Sie studieren nämlich Deutsch als Studienfach, wobei die Sprache nicht nur als Lernobjekt, sondern auch (oder vor allem) als Medium der Fachkommunikation fungiert. Insbesondere zählen die Sprech- und Prüfungsangst zu den Angstgefühlen, die im universitären fremdsprachlichen Sachfachunterricht in den ersten Semestern zum Vorschein kommen können. Dazu tragen sowohl die schulischen Vorerfahrungen der Lernenden als auch die Spezifik des Studierens bei. Es ist nämlich zu bemerken, dass die meisten Studierenden in eine ganz andere kommunikative Umgebung eintreten, in der die Kommunikationssituationen, -partner und auch -formen des Sprachgebrauchs für sie neu sind. In diesem Kontext müssen sich die Deutschkenntnisse der Erstsemester bewähren. Aus der Praxis der universitären Lehre wird auch deutlich, dass viele Studierende Zeit brauchen, um sich in der Gruppe durchzusetzen. So wird z. B. beobachtet, dass es Lernende gibt, die eine lange Zeit den anderen z.B. beim Sprechen zusehen, bevor sie selbst es wagen, das Wort zu ergreifen. Dieser Prozess dauert länger, wenn der Lernende bemerkt und subjektiv einschätzt, die anderen seien besser als er. Es ist sehr wichtig, diese Aspekte auch didaktisch zu konzipieren, indem die fremdsprachliche Kommunikation mit all ihren Problemen im Fachunterricht diskutiert wird. PHILLIPS (1992:20) weist darauf hin, dass die Lernenden sich erst einmal überzeugen sollten, dass Angstgefühle eine ganz normale Erscheinung seien, die für viele von ihnen ein Problem darstellten und auch den Lehrenden nicht unbekannt seien. Dabei ist vor allem die Einstellung der Lernenden zu Fehlern neu zu überdenken. Die Wichtigkeit der sprachlichen Korrektheit soll nicht in Frage gestellt werden, aber sie muss auch unter Berücksichtigung individueller Faktoren gesehen werden. Eine bloße Fehlerkorrektur genügt manchmal nicht. Des Weiteren sind falsche Überzeugungen der Lernenden, wie Sprachen gelernt werden, aus dem Weg zu räumen. Nicht selten herrscht unter den Lernenden immer noch die Überzeugung, Sprachenlernen setze Grammatik- und Wortschatzlernen voraus, ohne dabei auf solche Aspekte wie z.B. die Textgrammatik oder die grammatischlexikalische Pragmatik einzugehen. Fremdsprachliche Angst kann durch unterschiedliche Maßnahmen abgebaut oder zumindest vermindert werden. In erster Linie sollten die Lernenden ihre eigenen Lern- und Kommunikationsstile ken- 
Krzysztof Nerlicki

nenlernen. Auf ihrer Basis fällt auch die Wahl von geeigneten Lern- und Kommunikationsstrategien leichter. Die Lernenden sind imstande, Gründe zu nennen, die ihre Ängste verursachen. Wichtig ist also, den Lernenden zu ermöglichen, ihre Angstgefühle zu versprachlichen (z.B. in Gruppenarbeit oder in Form von Lerntagebüchern). ${ }^{22}$ Ein anderes Problem bilden mündliche Prüfungen, bei denen auf der Hand liegt, mit welchen Ängsten die Studierenden zu ringen haben. Es sollte auch im Interesse der fremdsprachlichen Praxis liegen, typische Prüfungssituationen, z. B. in Form von Rollenspielen, zu simulieren. Nicht selten hängt das positive Ergebnis einer Prüfung von der sprachlichen Bewältigung eines Problems ab.

\section{Ausblick}

Der Artikel versteht sich als Beitrag zur Diskussion über die Rolle individueller Faktoren beim Lernen einer Fremdsprache. Fremdsprachliche Angst bedarf einer besonderen Berücksichtigung, weil sie grundsätzlich negative Auswirkungen auf Lernprozesse haben kann. Die durchgeführte Analyse mit Hilfe eines Fragebogens zeigt die Einstellung der Lernenden zum untersuchten Problem. Es muss an dieser Stelle die Subjektivität eines solchen Unterfangens unterstrichen werden, weil die Lernenden nur ihre Meinungen geäußert haben, unabhängig davon, inwieweit diese mit der Wirklichkeit übereinstimmen (insbesondere bei Personen, die nicht gerne ihre Angstgefühle eingestehen). Wie aus dem Dargelegten zu erkennen ist, liegen die Mittelwerte der Ängstlichkeit bei den Germanistikstudierenden unter den bekannten anderen Ergebnissen. Das zeugt aber nicht davon, dass es im Studium keine ängstlichen Personen gibt. Immerhin basiert die fremdsprachliche Angst auf unterschiedlichen Vorerfahrungen der Lernenden, die ihre Ursachen in ihren Persönlichkeitsmerkmalen oder direkt im Fremdsprachenunterricht haben. Meines Wissens gibt es bisher keine Untersuchungen zur negativen Rolle des institutionellen Fremdsprachenunterrichts. Gemeint sind damit z. B. lehrerdominierende und durch institutionelle Maßnahmen geprägte Evaluationen, asymmetrische Redeanteile (Lehrerfragen - Lernerantworten) oder aber auf formale Richtigkeitsbestrebungen reduzierte Kontrollaufgaben. Es ist davon auszugehen, dass Ängstlichkeit, wenn auch nicht ganz, aber zumindest stark reduziert werden kann, wenn eine affektiv positive Umgebung geschaffen wird. Aus empirischer Perspektive wäre es auch interessant zu untersuchen, inwieweit eine bewusste, im Unterricht thematisierte Auseinandersetzung mit Angstgefühlen zu deren Abbau

22 Vgl. NERLICKI [in Vorbereitung]. 
beitragen könnte. In Anbetracht der gegenwärtig postulierten Lernerautonomie ist jede Form kooperativen Handelns, die die Lernprozesse fördert, begrüBenswert.

\section{Literatur}

AIDA, YUKIE (1994): Examination of Horwitz, Horwitz, and Cope's Construct of Foreign Language Anxiety: The Case of Students of Japanese. In: The Modern Language Journal 78:155-168.

ARNOLD, JANE (ed.) (1999): Affect in Language Learning. Cambridge.

AtAy, Derin / KURT, GÖKÇE (2006): Prospective Teachers and L2 Writing Anxiety. In: Asian EFL Journal 8:100-118.

Bailey, Kathleen M. (1983): Competitiveness and Anxiety in Adult Second Language Learning. In: SELIGER / LONG, 67-103.

Bailey, Philipp / Ownuegbuzie, Anthony J. / Daley, Christine E. (2000): Correlates of Anxiety at three Stages of the Foreign Language Learning Process. In: Journal of Language and Social Psychology 19:474-490.

Beushausen, Ulla (1996): Sprechangst. Erklärungsmodelle und Therapieformen. Opladen.

Chao, Chaun-Ta (2004): Foreign Language Anxiety and Emotional Intelligence: A Study of EFL Students in Taiwan: www.tamuk.edu (1.12.2006).

Davies, Alan / Elder, CATHERINe (eds.) (2004): Handbook of Applied Linguistics. London.

Duigunovic, Jelena M. (2006): Language Anxiety and Language Processing. In: EUROSLA Yearbook 6:191-212.

DÖRNYEI, ZOLTÁN / SKeHAN, PETER (2003): Individual Differences in Second Language Learning. In: DOUGHTY / LONG, 589-630.

Doughty, Catherine J. / Long, Michael H. (2003): The Handbook of Second Language Acquisition. Oxford.

EDMONDSON, WiLlis / HouSE, JULIANE (1993): Einführung in die Sprachlehrforschung. Tübingen/Basel.

ELLIS, RoD (2004): Individual Differences in Second Language Learning. In: DAVIES / ELDER, 525-551.

FISCHER, SYLVIA (2005): Sprechmotivation und Sprechangst im DaF-Unterricht. In: German as a foreign language 3: www.gfl-journal.com (1.12.2006).

GANSChOW, LeONORE / SPARKs, RichARD (1996): Anxiety about Foreign Language Learning among High School Women. In: The Modern Language Journal 80:199-212.

GARdner, Robert C. / Tremblay, PAul F. / MAsgoret, AnNe-Marie (1997): Towards a Full Model of Second Language Learning. An Empirical Investigation. In: The Modern Language Journal 81:344-362. 
Gregersen, Tammy S. (2005): Nonverbal Clues: Clues to the Detection of Foreign Language Anxiety. In: Foreign Language Annals 38:388-400.

Gregersen, TAmmy S. / Horwitz, Elaine K. (2002): Language Learning and Perfectionism: Anxious and Non-Anxious Language Learners' Reactions to their own oral Performance. In: The Modern Language Journal 86:562-570.

GROTJAHN, RÜDIGER (2004): Tests and Attitude Scales for the Year Abroad (TESTATT): Sprachlernmotivation und Einstellungen gegenüber Sprechern der eigenen und der fremden Sprache. In: Zeitschrift für Interkulturellen Fremdsprachenunterricht 9: www.ualberta.ca/ german/ejournal/ejournal.html (1.12.2006).

HACKFort, Dieter / SchwenkmeZger, Peter (1985): Angst und Angstkontrolle im Sport: Sportrelevante Ansätze und Ergebnisse theoretischer und empirischer Angstforschung. Köln.

Horwitz, Elaine K. (1988): The Beliefs about Language Learning of Beginning University Foreign Language Students. In: The Modern Language Journal 72:283-294.

- (1990): Attending to the affective domain in the foreign language classroom. In: MAGNAN, 15-33.

- (2000): It Ain't over 'til It's over: On Foreign Language Anxiety, First Language Deficits, and the Confounding of Variables. In: The Modern Language Journal 84:256259.

Horwitz, Elaine K. / Horwitz, Michael B. / Cope, Joann (1986): Foreign Language Classroom Anxiety. In: The Modern Language Journal 70:125-132.

LeAver, BetTy L. / Ehrman, Madeline / SHEKTMAN, BorIS (2005): Achieving Success in Second Language Acquisition. Cambridge.

MacIntyre, Peter D. / GARdner, Robert C. (1991): Investigating Language Class Anxiety Using the Focussed Essay Technique. In: The Modern Language Journal 75:296-304.

- (1994): The Subtle Effects of Language Anxiety on Cognitive Processing in the Second Language. In: Language Learning 44:283-305.

Macintyre, Peter D. / Noels, Kimberly A. / Clément, Richard (1997): Biases in Self-Ratings of Second Language Proficiency: The Role of Language Anxiety. In: Language Learning 47:265-287.

Magnan, Susan (ed.) (1990): Shifting the Instructional Focus to the Learner. Middlebury (Vt.).

MisSLER, BETTINA (1999): Fremdsprachenlernerfahrungen und Lernstrategien. Eine empirische Untersuchung. Tübingen.

NERLICKI, KRZYSZTOF: Ewaluacja osobistych procesów uczeniowych za pomocq dzienniczków. [Die Evaluation persönlicher Lernprozesse mit Hilfe von Lerntagebüchern]. [In Vorbereitung].

Ortega CEBreros, ANA Maria (2003): Measuring Language Anxiety perceived by Spanish University Students of English. In: Barcelona English Language and Literature Studies 12:50-62. 
OxFord, ReBecCA L. (1999): Anxiety and the Language Learner: New Insights. In: ARNOLD, 58-67.

Phillips, Elaine M. (1992): The Effects of Language Anxiety on Students' Oral Test Performance and Attitudes. In: The Modern Language Journal 76:14-26.

Saito, Yoshiko / Horwitz, Elaine K. / Garza, Thomas J. (1999): Foreign Language Reading Anxiety. In: The Modern Language Journal 83:202-218.

SELIGer, Herbert W. / LONG, Michael H. (eds.) (1983): Classroom oriented Research in Second Language Acquisition. Rowley.

SPARKS, Richard / GANSCHOw, LEONORE (1995): A Strong Inference Approach to Causal Factors in Foreign Language Learning: A Response to MacIntyre. In: The Modern Language Journal 79:235-244.

Stroud, Christopher / WeE, Lionel (2006): Anxiety and Identity in the Language Classroom. In: Regional Language Centre Journal 37:299-307.

WiLCZYŃSKA, WERONIKA (1999): Uczyć się czy być nauczanym? O autonomii w przyswajaniu języka obcego. [Lernen oder belehrt werden? Über die Autonomie bei der Aneignung einer Fremdsprache]. Warszawa/Poznań.

Young, Dolly Jesusita (1991): Creating a Low-Anxiety Classroom Environment: What Does Language Anxiety Research Suggest? In: The Modern Language Journal 75:426-439. 